\title{
THE GENERAL FORM OF THE SO-CALLED LAW OF THE ITERATED LOGARITHM
}

\author{
BY \\ W. FELLER
}

1. Introduction. The purpose of this paper is to sharpen Kolmogoroff's celebrated law of the iterated logarithm in various directions and to give best results. For the convenience of the reader, we shall link the statement of our problems with an account of the known results.

1.1. It seems that the law of the iterated logarithm traces its origin to a special problem in number theory. From the viewpoint of the theory of probability, as we shall see, this problem concerns only a very special case in which the general features are hardly visible. Nevertheless, this is the only case in which so far a final result has been achieved.

Let $p$ be a point of the interval $(0,1)$ and let $p=p_{1} p_{2} p_{3} \cdots$ be its binary expansion. (We shall be concerned with statements of the "almost everywhere" type, so ambiguity will do no harm.) Let

$$
X_{k}(p)=\left\{\begin{array}{lll}
+1 & \text { if } & p_{k}=1 \\
-1 & \text { if } & p_{k}=0
\end{array}\right.
$$

Then $S_{n}=X_{1}+\cdots+X_{n}$ is the excess frequency of occurrence of the digit 1 among the first $n$ places in the expansion of $p$. The strong law of large numbers (Borel, Cantelli) asserts that almost everywhere $S_{n}=o(n)$. The following enumeration of sharper results indicates the historic development of the problem. It is, of course, understood that all assertions hold true only for almost all points $p$.

(i) Hausdorff, [6], 1913:

$$
S_{n}=o\left(n^{1 / 2+\epsilon}\right), \quad \epsilon>0 .
$$

(ii) Hardy-Littlewood, [4], 1914:

$$
S_{n}=O\left((n \log n)^{1 / 2}\right) \text {. }
$$

(iii) Steinhaus, [16], 1922:

$$
\limsup _{n \rightarrow \infty} S_{n} /(2 n \log n)^{1 / 2} \leqq 1 .
$$

(iv) Khintchine, [7], 1923:

$$
S_{n}=O\left((n \log \log n)^{1 / 2}\right) .
$$

Presented to the Society, February 27, 1943; received by the editors March 15, 1943. 
(v) Khintchine, [8], 1924:

$$
\limsup _{n \rightarrow \infty} S_{n} /(2 n \log \log n)^{1 / 2}=1 \text {. }
$$

In formulating newer results we shall, following $\mathrm{P}$. Lévy, say that a function $\phi(t)$ belongs to the upper class $(\in U)$ if, for almost all $p$, there exist only finitely many $n$ such that $S_{n}>n^{1 / 2} \phi(n)$; and we shall say that $\phi(t)$ belongs to the lower $\operatorname{class}\left({ }^{1}\right)(\in \mathcal{L})$ if, for almost all $p$, the inequality $S_{n}>n^{1 / 2} \phi(n)$ is satisfied for infinitely many $n$. Khintchine's result $(v)$ then reads:

$$
\begin{aligned}
a(2 \log \log t)^{1 / 2} \in \mathcal{V} & \text { if } \quad a>1, \\
\in \mathcal{L} \text { if } & a<1 .
\end{aligned}
$$

(vi) P. Lévy, [11], 1933:

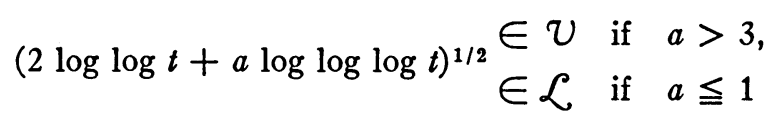

(with a gap for $1<a \leqq 3$ ).

(vii) Kolmogoroff-Erdös $\left({ }^{2}\right)$, (1937-1942): If $\phi(t)$ is non-decreasing, then $\left({ }^{8}\right)$

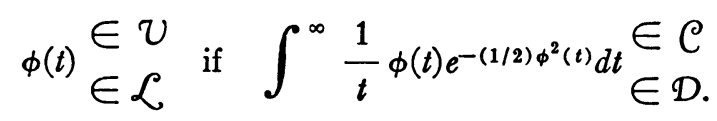

The last result contains, of course, the preceding ones. It gives, in a most elegant way, a complete solution for the binomial case with probabilities $1 / 2$. It is our purpose to find a similar solution for arbitrary independent random variables, that is to say, for arbitrary bounded real functions $X_{k}$ in arbitrary spaces. As a matter of fact, the above solution is so specialized that it fails already in the next simplest problem concerning the frequency of occurrence of digits in the ordinary decimal expansion.

1.2. For a general formulation of the problem consider an infinite sequence of spaces $\left\{E_{k}\right\}, k=1,2, \cdots$, in which probability-measures are defined( $\left.{ }^{4}\right)$. Let $E=E_{1} \times E_{2} \times \cdots \times E_{k} \times \cdots$ be their infinite cómbinatorial product

(1) According to the law of 0 or 1 each $\phi(t)$ belongs to one of these classes. We shall not use this result in the sequel.

(2) Kolmogoroff's result has been stated, without proof, in P. Lévy's book [12]. Erdös [2] proved the result completely. Before, J. Ville [17] had proved that convergence of the integral is a sufficient condition for the upper class. It is the necessity of this condition which presents the real difficulties. Most of the results of the present paper have been obtained before publication of Erdös' paper.

(3) Here and in the following $\mathcal{C}$ and $\mathcal{D}$ stand for "converges" and "diverges," respectively.

(4) In each $E_{k}$ there is a Borel family of sets $\mathfrak{F}_{k}$ such that $E_{k} \in \mathfrak{F}_{k}$; on $\mathfrak{F}_{k}$ we have a completely additive set-function $\operatorname{Pr}(\Gamma)$ with $0 \leqq \operatorname{Pr}(\Gamma) \leqq 1$ and $\operatorname{Pr}\left(E_{k}\right)=1$. 
and let a probability-measure in $E$ be defined in the customary manner( $\left(^{(5)}\right.$. Let $X_{k}=X_{k}\left(p_{k}\right)$ be a real-valued function of a point $p_{k}$ varying in $E_{k}$; then $\left(^{6}\right)$

$$
S_{n}=X_{1}+\cdots+X_{n}
$$

is a function of the point $p=p_{1} \times p_{2} \times \cdots \in E$. Let

$$
V_{k}(x)=\operatorname{Pr}\left\{X_{k} \leqq x\right\}
$$$$
-\infty<x<\infty
$$

be the distribution function of $X_{k}$. Then the distribution function $F_{n}(x)$ of $S_{n}$ is found from

$$
F_{n+1}(x)=\int_{-\infty}^{+\infty} F_{n}(x-y) d V_{n+1}(y) .
$$

We shall suppose that

$$
\sigma_{k}^{2}=\int_{-\infty}^{+\infty} x^{2} d V_{k}(x)
$$

exists; replacing, if necessary, $X_{k}$ by $X_{k}+c_{k}$ we can without loss of generality assume that

$$
\int_{-\infty}^{+\infty} x d V_{k}(x)=0, \quad k=1,2, \cdots .
$$

The second moment of $F_{n}(x)$ is

(5) We shall actually consider only sets of the type $\Gamma=\cdots \times \Gamma_{i_{1}} \times \cdots \times \Gamma_{i_{2}} \times \cdots$ $\times \Gamma_{i_{n}} \times \cdots$, where $\Gamma_{i_{k}} \in \mathfrak{F}_{i_{k}}$ and the dots stand for $E_{n}$. Of course, $\operatorname{Pr}(\Gamma)=\operatorname{Pr}\left(\Gamma_{i_{1}}\right) \cdots \operatorname{Pr}\left(\Gamma_{i_{n}}\right)$. If $\Gamma=\left(\Gamma_{i_{1}}, \cdots, \Gamma_{i_{n}}\right)$ and $\Lambda=\left(\Lambda_{i_{1}}, \cdots, \Lambda_{i_{m}}\right)$ are two such sets, they will be called $i n d e p e n d e n t$, if $i_{\rho} \neq j_{\sigma}$; then $\operatorname{Pr}(\Gamma \Lambda)=\operatorname{Pr}(\Gamma) \operatorname{Pr}(\Lambda)$. For our purposes it is sufficient to have a measure-theory for finite combinatorial products and the corresponding notion of sets of measure zero in the infinite product space.

(8) In this formulation it becomes clear that, as far as mathematics is concerned, we are dealing with a problem in real function theory in product spaces. The sense, and importance, of our investigation for probability and statistics is, it is hoped, obvious. The subscript $k$ stands, of course, for the order number of the experiment or observation. $E_{k}$, the $k$ th label space, is the abstract expression for the set of all thinkable results of the $k$ th experiment, each result being represented by a point $p_{k} \in E_{k}$. (In practice these results will be: head-or-tail; the position of the roulette; the position of a particle subjected to diffusion or, more generally, the phase-space in physics; or the complex composition with respect to age, claims, risk, and so on, of the stock of policies of an insurance company.) $X_{k}$ will be the gain or any other characteristic in the $k$ th experiment and we are interested in the fluctuations of the accumulated value of that characteristic after $n$ experiments. The epistemological problem of what "probability" means has no more to do with theory or practice than the corresponding problem of space conception in geometry.

It may be remarked that we do not attach any importance to the possibility that the $E_{k}$ are not similar to each other. The fact of the situation is that the structure of $E_{k}$ simply nowhere appears. 


$$
s_{n}^{2}=\sigma_{1}^{2}+\cdots+\sigma_{n}^{2} .
$$

For obvious reasons only the case where

$$
s_{n} \rightarrow \infty
$$

presents a problem.

We shall, following P. Lévy, say that a sequence $\left\{\phi_{n}\right\}$ belongs to the lower class $\left(\in \mathcal{L}\right.$ ) (with respect to $\left\{X_{k}\right\}$ ), if, for almost all $p \in E$, there exist infinitely many $n$ such that

$$
S_{n}>s_{n} \phi_{n}
$$

and to the upper class $(\in \mathcal{U})$ if, for almost all $p \in E$, there exist only finitely many $n$ such that (1.7) holds. (It will be noticed that Theorem 8 actually contains a sharpening of the notion of upper class: in defining the upper class we could as well replace (1.7) by (2.19).)

1.3. The law of the iterated logarithm states: if each $X_{k}$ is bounded and

$$
\text { l.u.b. }\left|X_{k}\right|=o\left(s_{n} / \log _{2}^{1 / 2} s_{n}\right)
$$

uniformly in $k=1,2, \cdots, n$, then

$$
\begin{aligned}
\left(a \log _{2} s_{n}\right)^{1 / 2} & \in \mathcal{V} \text { if } a>2, \\
\in \mathcal{L} \text { if } & a<2 .
\end{aligned}
$$

This is the most general result known; it was proved by A. Kolmogoroff [10], after important special cases had been solved by A. Khintchine [9]. A new proof of (1.9) under more restrictive conditions than (1.8) has been given by $P$. Lévy [12]. Under much stronger conditions Cantelli [1] has proved a result which is sharper than (1.9): suppose that for some $c>2$

$$
\int_{-\infty}^{+\infty}|x|^{d} d V_{k}(x), \quad k=1,2, \cdots \text {, }
$$

exists, and that uniformly in $k$

$$
\int_{-\infty}^{+\infty} x^{2} d V_{k}(x) \geqq a_{0}, \quad \int_{-\infty}^{+\infty}|x|^{c} d V_{k}(x) \leqq a_{1} ;
$$

then (even not assuming (1.8))

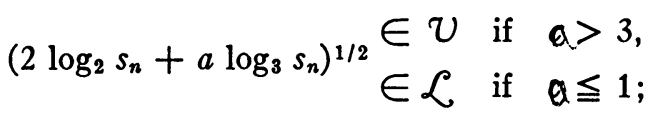

the gap between 1 and 3 is left open. (Cf. above P. Lévy's result vi.)

There is a most surprising result due to Marcinkiewicz and Zygmund [15] which is important for the proper understanding of our problem. They showed 
that if (1.8) be replaced by the only slightly less restrictive condition:

$$
\text { l.u.b. }\left|X_{k}\right|<\epsilon\left(s_{n} / \log _{2}^{1 / 2} s_{n}\right)
$$

( $\epsilon$ arbitrary but fixed), then (1.9) becomes false. In fact, they constructed a sequence $\left\{X_{k}\right\}$ satisfying (1.10) but for which

$$
\limsup _{n \rightarrow \infty}\left(S_{n} / s_{n}\left(2 \log _{2} s_{n}\right)^{1 / 2}\right)<1 .
$$

1.4. In the present paper we shall give necessary and sufficient conditions for a sequence $\left\{\phi_{n}\right\}$ to belong to the upper or lower class. It will be seen that our criterion assumes the simple form of Kolmogoroff's criterion (vii) if the $\left\{X_{k}\right\}$ are subject to the condition( $\left(^{7}\right)\left|X_{k}\right|=O\left(s_{n} / \phi_{n}^{3}\right)$ (Theorems 2 and 6). As this condition is gradually relaxed the criterion assumes an increasingly complicated form. Thus, if $\left|X_{k}\right|=O\left(s_{n} / \phi_{n}^{2}\right)$ the criterion will involve third moments of the distribution functions $V_{k}(x)$; this implies a remarkable lack of symmetry, since now $\left\{\phi_{n}\right\}$ may belong to the upper class for the sequence $\left\{X_{k}\right\}$ and to the lower class for $\left\{-X_{k}\right\}$ (Theorem 3). If it is assumed only that $\left|X_{k}\right|=O\left(s_{n} / \phi_{n}^{5 / 3}\right)$ the fourth moments of $\left\{V_{k}(x)\right\}$ will be of influence also, and so on (Theorem 5). Finally, when the upper bounds of $\left|X_{k}\right|$ are of the order $C\left(s_{n} / \phi_{n}\right)$ all moments of $\left\{V_{k}(x)\right\}$ enter into consideration; when $C$ is very small, the dominant influence will still be exercised by the second moments; however, as $C$ increases, the dominant role gradually shifts to moments of an increasingly higher order. It is thus seen that if the upper bounds of $\left|X_{k}\right|$ increase faster than $s_{n} / \phi_{n}$, we shall be confronted with an altogether new situation and any criterion would necessarily be of a quite different form $\left(^{8}\right)$. This domain is not investigated in the present paper. The criterion given in Theorem 1 still covers the case (1.10) (with $\epsilon=1 / 200$ ) and thus explains the phenomenon detected by Marcinkiewicz and Zygmund by giving the precise limit between the upper and the lower class.

It will be seen that the present paper considers only individually bounded variables $X_{k}$. It is hardly necessary to point out that most sequences which occur in standard applications can be reduced to this case by the customary method of equivalent sequences (truncating the $X_{k}$ at values $M_{k}$ such that $\sum \operatorname{Pr}\left\{\left|X_{k}\right|>M_{k}\right\}$ converges). This method makes it evident, for example, that Kolmogoroff's law of the iterated logarithm is applicable if

$\left.{ }^{7}\right)$ For a proper understanding of the theorems (as well as for a comparison with Kolmogoroff's condition (1.8)) it should be noticed that we shall be interested usually in sequences $\left\{\phi_{n}\right\}$ near the borderline between the upper and the lower class. That is to say, roughly speaking $\phi_{n}$ will increase like $\left(2 \log \log s_{n}\right)^{1 / 2}$.

${ }^{8}$. This observation is also borne out by results of P. Lévy [13] and Marcinkiewicz [15], who investigated the analogue to the law of the iterated logarithm in the special case where $c x^{-a} \leqq 1-V_{k}(x) \leqq C x^{-a}$, with $0<a<1$ and two constants $C>c>0$. 
$V_{k}(x) \equiv V(x)$ where $V(x)$ has a finite moment of order $2+\epsilon>2\left({ }^{9}\right)$. Actually it is possible to extend also the exact criteria of the present paper to very large classes of sequences $\left\{X_{k}\right\}$ of unbounded variables. An example of such a class (containing most usual sequences) is given in the appendix. In the construction it seemed desirable to aim at greater simplicity rather than at greatest possible generality.

Our proofs are direct and do not presuppose previous work on the subject. Beyond standard tools of measure theory we shall use only a theorem describing the behavior of $F_{n}(x)$ for large $x$; this theorem is a sharpening of the classical central limit theorem of probability and has been proved in a previous paper $\left({ }^{10}\right)$.

2. Theorems. Throughout the sequel $X_{k}$ will stand for a real function in $E_{k}$ (see $\S 1.2) ; S_{n}$ is defined by (1.1) and $V_{k}(x)$ by (1.2); it shall be assumed that (1.3), (1.4), and (1.6) hold. Moreover we shall suppose that there exists a sequence $\lambda_{n} \downarrow 0$ such that ${ }^{(11)}$

$$
\text { l.u.b. }\left|X_{n}\right|<\lambda_{n} s_{n} \text {, }
$$

where $s_{n}$ is defined by (1.5). Of the sequence $\left\{\phi_{n}\right\}$ we shall assume that $\left.{ }^{12}\right)$

$$
2<\phi_{1} \leqq \phi_{2} \leqq \phi_{3} \leqq \cdots .
$$

2.1. For the most general case of our criterion we shall require a function $Q_{n}(x)$ which regulates the behavior of $F_{n}\left(x s_{n}\right)$ for large $x$. Define, in a formal way, quantities $\gamma_{k, \nu}$ and $\Gamma_{n, \nu}$ by

and

$$
\log \int_{-\infty}^{+\infty} e^{h y} d V_{k}(y)=\sum_{\nu=2}^{\infty} \gamma_{k, \nu} \frac{h^{\nu}}{\nu !}
$$

(9) This seems to have escaped the attention of Hartman and Wintner [5], in their strong remarks about the inapplicability of Kolmogoroff's law to sequences occurring in standard applications. Actually it suffices to suppose that $\int_{-\infty}^{+\infty} x^{2}|\log | x||{ }^{1+\epsilon} d V(x)=A$ is finite for some $\epsilon>0$. Then $\sum_{k=100}^{\infty} \int|x|>k^{1 / 2} / \log \log k d V(x)<A \sum_{k=100}^{\infty}\left(\log \log s_{k}\right)^{4} / k \log ^{1+\epsilon} k$ converges; the truncated variables obey the law of the iterated logarithm and the variances of their sums are asymptotically equivalent to $s^{2}$. Hartman and Wintner [5] have devised a slightly more efficient method of truncation which shows that it is sufficient to suppose that $\int_{-\infty}^{+\infty} x^{2} d V(x)$ exists. It does not follow that in this case also our criteria hold.

(10) Feller [3], in the sequel referred to as L.T. The present paper should be readable without knowledge of L.T.

(i1) The monotony of $\left\{\lambda_{n}\right\}$ is used exclusively to assure that 1.u.b. $\left|X_{k}\right|<\lambda_{n} s_{n}$ for $k=1,2, \cdots, n$.

(12) It seems only natural to assume monotony of $\left\{\phi_{n}\right\}$. The restriction is unessential and the proofs become even slightly simpler if, instead, it is assumed that the variation of $\left\{\phi_{n}\right\}$ in any interval is small as compared with $1 / \phi_{n}$. However, abrupt changes of $\phi_{n}$ are interesting and considered in the sequel. If $\left\{\phi_{n}\right\}$ remains bounded, the theorems become trivial. The assumption $\phi_{n}>2$ is convenient and presents no loss of generality. 


$$
\Gamma_{n, \nu}=\sum_{k=1}^{n} \gamma_{k, \nu}
$$

( $\gamma_{k, 1}=0$ by (1.4); only small values of $h$ will be considered and it is understood that the $\gamma_{k, v}$ are real). Then

$$
\frac{x^{2}}{2}\left\{1+Q_{n}(x)\right\} \equiv \sum_{\nu=2}^{\infty} \Gamma_{n, \nu} \frac{\nu-1}{\nu !} h^{\nu}
$$

where $h=h(x)$ is the inverse function of

$$
x=\frac{1}{s_{n}} \sum_{\nu=2}^{\infty} \Gamma_{n, \nu} \frac{h^{\nu-1}}{(\nu-1) !} .
$$

It has been shown in L.T. that, if $x \lambda_{n}<1 / 12, Q_{n}(x)$ is an analytic function

$$
Q_{n}(x)=\sum_{\nu=1}^{\infty} q_{n, \nu} x^{\nu}
$$

and

$$
\left|q_{n, \nu}\right|<(1 / 7)\left(12 \lambda_{n}\right)^{\nu}
$$

In general, $q_{n, \nu}$ depends on the first $\nu+2$ moments of $V_{1}(x), \cdots, V_{n}(x)$,

$$
b_{k, \rho}=\int_{-\infty}^{+\infty} x^{\rho} d V_{k}(x), \quad \rho=1, \cdots, \nu+2 .
$$

It follows from the main theorem of L.T. that, under the conditions of the present paper, the ratio of $F\left(s_{n} \phi_{n}\right)$ to $\phi_{n}^{-1}\left\{\exp -(1 / 2) \phi_{n}^{2}\left[1+Q_{n}\left(\phi_{n}\right)\right]\right\}$ remains between two positive constants. This explains the importance of the function $Q(x)$ for the present investigation.

2.2. In the following theorems the main conditions $((2.6),(2.8),(2.12)$, and so on) depend on the sequence $\left\{\phi_{n}\right\}$ which is to be tested. Although these conditions seem most natural, it is sometimes more advantageous to replace them by conditions which do not explicitly depend on $\left\{\phi_{n}\right\}$. Such alternative conditions will be formulated in Theorem 11 .

THEOREM 1. If

$$
\lambda_{n} \leqq 1 / 200 \phi_{n}
$$

then $\left\{\phi_{n}\right\} \in \mathcal{U}(\mathcal{L})$ if, and only if $\left({ }^{13}\right)$,

$$
\sum_{n} \frac{\sigma_{n}^{2}}{s_{n}^{2}} \phi_{n} \exp \left\{-(1 / 2) \phi_{n}^{2}\left[1+Q_{n}\left(\phi_{n}\right)\right]\right\} \in \mathcal{C}(\mathcal{D}) .
$$

(13) Cf. footnote 3. 
Postponing the proof of the theorem we mention a few corollaries.

THEOREM 2. If

$$
\lambda_{n}=O\left(1 / \phi_{n}^{3}\right)
$$

then $\left\{\phi_{n}\right\} \in \mathcal{U}(\mathcal{L})$ if, and only if,

$$
\sum_{n} \frac{\sigma_{n}^{2}}{s_{n}^{2}} \phi_{n} e^{-(1 / 2) \phi_{n}^{2}} \in \mathcal{C}(\mathcal{D})
$$

In fact, using (2.4) it is seen that (2.8) implies $\phi_{n}^{2} Q_{n}\left(\phi_{n}\right)=O(1)$ and Theorem 2 follows from Theorem 1. Similarly one proves another corollary:

TheOREM 3. If

$$
\lambda_{n}=O\left(1 / \phi_{n}^{2}\right)
$$

then $\left\{\phi_{n}\right\} \in U(\mathcal{L})$ if, and only if,

$$
\sum_{n} \frac{\sigma_{n}^{2}}{s_{n}^{2}} \phi_{n} \exp \left\{-(1 / 2) \phi_{n}^{2}-\left(\phi_{n}^{3} / 6 s_{n}^{3}\right) \sum_{k=1}^{n} b_{k, 3}\right\} \in \mathcal{C}(\mathcal{D})
$$

where $b_{k, 3}$ is defined by (2.5).

In the case of symmetric variables $X_{k}$ the moments $b_{k, 3}$ vanish. Therefore:

ThEOREM 4. If $1-V_{k}(x)=V_{k}(-x)$ and (2.10) holds, then $\left\{\phi_{n}\right\} \in \mathcal{U}(\mathcal{L})$ if, and only if, the series (2.9) converges (diverges).

In general we have in the same way the following theorem.

THEOREM 5. If, for some integer $\rho>1$,

$$
\lambda_{n}=O\left(1 / \phi_{n}^{(\rho+2) / \rho}\right)
$$

then $\left\{\phi_{n}\right\} \in \mathcal{U}(\mathcal{L})$ if, and only if,

$$
\sum_{n} \frac{\sigma_{n}^{2}}{s_{n}^{2}} \phi_{n} \exp \left\{-(1 / 2)\left[\phi_{n}^{2}+q_{n, 1} \phi_{n}^{3}+\cdots+q_{n, \rho-1} \phi_{n}^{\rho+1}\right]\right\} \in \mathcal{C}(\mathcal{D}) ;
$$

thus the behavior depends only on the first $\rho+1$ moments of $\left\{V_{k}(x)\right\}$. We have in particular

$$
\begin{aligned}
q_{n, 1} & =\frac{1}{3 s_{n}^{3}} \sum_{k=1}^{n} b_{k, 3} \\
q_{n, 2} & =\frac{1}{12 s_{n}^{4}} \sum_{k=1}^{n} b_{k, 4}-\frac{1}{4 s_{n}^{4}} \sum_{k=1}^{n} b_{k, 2}^{2}-\frac{1}{4 s_{n}^{6}}\left(\sum_{k=1}^{n} b_{k, 3}\right)^{2}
\end{aligned}
$$

In $\$ 7$ it will be proved that the criterion of Theorem 2 can be reformulated 
in a way analogous to Kolmogoroff's criterion in the binomial case as follows.

THEOREM 6. If $\phi(t) \uparrow$ and

$$
\lambda_{n}=O\left(1 / \phi^{3}\left(s_{n}^{2}\right)\right)
$$

then $\left\{\phi\left(s_{n}^{2}\right)\right\} \in \mathcal{U}(\mathcal{L})$ if, and only if,

$$
\int^{\infty} \frac{1}{t} \phi(t) e^{-(1 / 2) \phi^{2}(t)} d t \in \mathcal{C}(\mathcal{D}) .
$$

The connection between our theorems and the law of the iterated logarithm becomes more apparent in the following theorem.

\section{THEOREM 7. If either}

$$
\lambda_{n}=O\left(1 / \log _{2}^{3 / 2} s_{n}\right),
$$

or $V_{k}(-x)=1-V_{k}(x)$ and

$$
\lambda_{n}=O\left(1 / \log _{2} s_{n}\right),
$$

then

$$
\phi_{n} \equiv\left\{2 \log _{2} s_{n}^{2}+3 \log _{3} s_{n}^{2}+2 \log _{4} s_{n}^{2}+\cdots\right.
$$

$$
\left.+2 \log _{\rho-1} s_{n}^{2}+(2+\delta) \log _{\rho} s_{n}^{2}\right\}^{1 / 2} \in V(\mathcal{L})
$$

if, and only if,

$$
\delta>0(<0) .
$$

This theorem is actually a simple corollary to Theorems 2 and 4 . For the proof it suffices to note that, with the definition (2.18), $\phi_{n}=\left(2 \log _{2} s_{n}\right)^{1 / 2}+o(1)$, so that (2.16) and (2.17) are equivalent to (2.8) and (2.10), respectively. Thus the criterion (2.9) is applicable. However,

$$
\sum \frac{\sigma_{n}^{2}}{s_{n}^{2}} \phi_{n} e^{-(1 / 2) \phi_{n}^{2}}=(2)^{1 / 2} \sum \frac{\sigma_{n}^{2}(1+o(1))}{s_{n}^{2} \log s_{n}^{2} \log _{2} s_{n}^{2} \cdots \log _{\rho}^{1+\delta / 2} s_{n}^{2}} ;
$$

now $\sum \sigma_{n}^{2}$ diverges, and therefore $\left({ }^{14}\right)$ the above series converges if, and only if, $\delta>0$.

We shall prove the following sharpening of the notion of lower class, which holds true in all cases considered in this paper.

(14) This follows easily from theorems of Abel-Dini and of Cesàro. See, for example, K. Knopp, Theory and applications of infinite series, London-Glasgow, Blackie and Son, 1928 , pp. $292 \mathrm{ff}$. Theorem 7 is, of course, an immediate consequence also of Theorem 6. Specialized to.the symmetric binomial case it becomes a consequence of the theorem of KolmogoroffErdös. It should be noted that the terms $s_{n}^{2}$ in (2.18) can be replaced by $s_{n}$. 
THEOREM 8. Under the condition (2.6) of Theorem 1, if $\left\{\phi_{n}\right\} \in \mathcal{L}$ then for any couple of constants $\alpha<\beta$ and at almost all points, the inequality

$$
s_{n}\left(\phi_{n}+\alpha / \phi_{n}\right)<S_{n}<s_{n}\left(\phi_{n}+\beta / \phi_{n}\right)
$$

will be satisfied for infinitely many $n$.

Hence: If $\left\{\phi_{n}\right\} \in \mathcal{L}$ also $\left\{\phi_{n}+\alpha / \phi_{n}\right\} \in \mathcal{L}$.

Besides the criterion of Theorem 1 we shall prove the following (less handy) criterion :

THEOREM 9. Under the condition (2.6) of Theorem 1, the sequence $\left\{\phi_{n}\right\} \in U(\mathcal{L})$ if, and only if, for any sequence of integers $\left\{n_{k}\right\}$ for which

$$
s_{n_{k}}^{2}\left(1+a / \phi_{n_{k}}^{2}\right)<s_{n_{k+1}}^{2}<s_{n_{k}}^{2}\left(1+b / \phi_{n_{k}}^{2}\right),
$$

where $a$ and $b$ are constants, the series

$$
\sum \operatorname{Pr}\left\{s_{n_{k}}>s_{n_{k}} \phi_{n_{k}}\right\} \in \mathcal{C}(\mathcal{D}) \text {. }
$$

We mention also the following corollary to Theorem 1 , which follows easily by means of (2.4).

THEOREM 10. Under the condition (2.6) of Theorem 1 if

$$
\sum_{n} \frac{\sigma_{n}^{2}}{s_{n}^{2}} \phi_{n} e^{-(1 / 2) \phi_{n}^{2}} \in \mathcal{C},
$$

the sequence

$$
\left\{\phi_{n}+\lambda_{n} \phi_{n}^{2}\right\} \in V
$$

if (2.22) diverges,

$$
\left\{\phi_{n}-\lambda_{n} \phi_{n}^{2}\right\} \in \mathcal{L}
$$

Finally, we give an alternative form for the conditions of the preceding theorems. Its usefulness will be exemplified by the results of the appendix.

THEOREM 11. The criterion of Theorem 1 remains valid if the condition (2.6) is replaced by

$$
\lambda_{n} \leqq 1 / 200\left(\log \log s_{n}\right)^{1 / 2} .
$$

$A$ similar remark applies to Theorems 2-6. If (2.25) is satisfied, the sequence $\left\{\phi_{n}\right\}$ belongs to the same class as the sequence $\left\{\psi_{n}\right\}$ defined by

$$
\psi_{n}=\min \left\{\phi_{n}, 2\left(\log \log s_{n}\right)^{1 / 2}\right\} .
$$

For a generalization of the preceding results to the case of unbounded variables $\left\{X_{n}\right\}$ the reader is referred to the appendix. 
2.3. Remarks. The following remarks are either obvious or easily verified. They will not be used in the sequel, and their detailed proof can therefore be left to the reader.

1. Theorem 8 contains a best result in two directions. The assertion that $\left\{\phi_{n}\right\}$ and $\left\{\phi_{n}+\right.$ const $\left./ \phi_{n}\right\}$ belong to the same class cannot be improved. First, if $\left\{X_{n}\right\}$ is a given sequence and $a_{n} \rightarrow \infty$, it is possible to find a sequence such that $\left\{\phi_{n}\right\} \in \mathcal{L}$ but $\left\{\phi_{n}+a_{n} / \phi_{n}\right\} \in \mathcal{U}$ (cf. Erdös [2] in the case of the binomial distribution with equal probabilities). More interesting is that to any given $\left\{\phi_{n}\right\}$ and $a_{n} \rightarrow \infty$ it is possible to find a sequence $\left\{X_{n}\right\}$ such that $\left\{\phi_{n}\right\} \in \mathcal{L}$ but $\left\{\phi_{n}+a_{n} / \phi_{n}\right\} \in U$. The fact that $\left\{\phi_{n}\right\}$ and $\left\{\phi_{n}+a / \phi_{n}\right\}$ belong to the same class is easily established independently of the previous results. In fact, it is sufficient to notice that $\left|\left(\phi_{n}+a / \phi_{n}\right)^{2} Q_{n}\left(\phi_{n}+a / \phi_{n}\right)-\phi_{n}^{2} Q_{n}\left(\phi_{n}\right)\right|=O(1)$, and this is readily proved using (2.4).

2. If $\lambda_{n}=O\left(1 / \phi_{n}^{3}\right)$ then $\left\{\phi_{n}\right\}$ is of the same class with respect to the sequence $\left\{X_{n}\right\}$ and $\left\{-X_{n}\right\}$. This is a best result. If $a_{n} \rightarrow \infty$ is an arbitrary sequence it is possible to find a sequence $\left\{X_{n}\right\}$ with $\lambda_{n}=O\left(a_{n} / \phi_{n}^{3}\right)$ such that $\left\{\phi_{n}\right\} \in \mathcal{U}$ for $\left\{X_{n}\right\}$ but $\left\{\phi_{n}\right\} \in \mathcal{L}$ for $\left\{-X_{n}\right\}$.

3. It has been conjectured by P. Lévy $[12$, p. 266] that Kolmogoroff's criterion (Theorem 6) is applicable for any uniformly bounded sequence $\left\{X_{k}\right\}$. He put the problem of finding the weakest conditions on $\left\{\lambda_{n}\right\}$ under which the theorem would hold. Theorem 6 gives the best result. If $\lambda_{n} \phi_{n}^{3} \rightarrow \infty$, it is possible to find a sequence $\left\{X_{n}\right\}$ such that the criterion of Theorems 2 and 6 becomes false.

4. It will be noticed that the condition (2.16) is sharper than Kolmogoroff's condition (1.8). However, (2.16) is the best condition. If $\lambda_{n} \log _{2}^{3 / 2} s_{n} \rightarrow \infty$ it is possible to construct a sequence $\left\{X_{n}\right\}$ such that (2.18) does not hold.

5. It is interesting to compare Theorem 10 with the classical law of the iterated logarithm, and with our theorems. If $\lambda_{n}=O\left(1 / \phi_{n}^{3}\right)$ then the sequences $\left\{\phi_{n} \pm \lambda_{n} \phi_{n}^{3}\right\}$ obviously belong to the same class, and Theorem 10 reduces to Theorem 1. However, as the order of magnitude of $\left\{\lambda_{n}\right\}$ increases, Theorem 10 becomes less and less sharp. With the law of the iterated logarithm one knows only that $\lambda_{n}=o\left(1 / \phi_{n}\right)$; hence the assertion relates only to sequences of the form $\left\{\phi_{n} \pm \epsilon \phi_{n}\right\}$. When $\lambda_{n}=O\left(1 / \phi_{n}\right)$ even this breaks down, which explains the behavior of the counter-example constructed by Marcinkiewicz and Zygmund.

6. It is hardly necessary to point out that the constant $1 / 200$ in Theorem $1(2.6)$ is convenient but purely arbitrary. Actually $1 / 12$ would have been sufficient (except for minor complications). There is no best constant, as the radius of convergence of $Q_{n}(x)$ depends on the sequence $\left\{X_{n}\right\}$.

3 . The sequence $\left\{n_{k}\right\}$. We proceed first to establish the existence of sequences of the kind occurring in Theorem 9: If condition (2.6) is satisfied then to any constants $a, b$ with 


$$
0<a<(99 / 100) b, \quad b>1 / 100,
$$

there exists a sequence $\left\{n_{k}\right\}$ such that (2.20) holds. Obviously $n_{k} \rightarrow \infty$.

The proof is very simple. Since, by assumption, $s_{n} \rightarrow \infty$ we can choose $n_{1}$ arbitrarily and define a sequence $\left\{n_{k}\right\}$ by the recurrence relations

$$
\begin{aligned}
s_{n_{k+1}}^{2} & \leqq s_{n_{k}}^{2}\left(1+b / \phi_{n_{k}}^{2}\right), \\
s_{n_{k+1}+1}^{2} & >s_{n_{k}}^{2}\left(1+b / \phi_{n_{k}}^{2}\right) .
\end{aligned}
$$

Then by (1.5), (2.1), (2.6), and (2.2)

$$
\begin{aligned}
s_{n_{k+1}+1}^{2} & =s_{n_{k+1}}^{2}+\sigma_{n_{k+1}+1}^{2} \leqq s_{n_{k+1}}^{2}+10^{-4} \bar{\phi}_{n_{k+1}+1}^{2} s_{n_{k+1}+1}^{2} \\
& \leqq s_{n_{k+1}}^{2}+10^{-4} \bar{\phi}_{n_{k}}^{2} s_{n_{k+1}+1}^{2},
\end{aligned}
$$

or, using (3.3) and (3.1),

$$
\begin{aligned}
s_{n_{k+1}}^{2} & \geqq s_{n_{k+1}+1}^{2}\left(1-10^{-4}-\phi_{n_{k}}^{-2}\right)>s_{n_{k}}^{2}\left(1+b / \phi_{n_{k}}^{2}\right)\left(1-10^{-4} \phi_{n_{k}}^{-2}\right) \\
& \geqq s_{n_{k}}^{2}\left(1+a / \phi_{n_{k}}^{2}\right) .
\end{aligned}
$$

In the following $\left\{n_{k}\right\}$ shall stand for an arbitrary, but fixed, sequence satisfying (2.20). We shall write

(3.4) $S_{n_{k}}=S_{k}^{\prime}, \quad s_{n_{k}}=s_{k}^{\prime}, \quad \phi_{n_{k}}=\phi_{k}^{\prime}, \quad Q_{n_{k}}(x)=Q_{k}^{\prime}(x), \quad \lambda_{n_{k}}=\lambda_{k}^{\prime}$.

This will greatly simplify the notation, as we shall require a subsequence of $\left\{n_{k}\right\}$ and, in turn, a subsequence of it.

4. Lemma 1. Suppose that condition (2.6) is satisfied and that

$$
\sum \operatorname{Pr}\left\{S_{k}^{\prime}>s_{k}^{\prime} \phi_{k}^{\prime}\right\} \in \mathcal{D} \text {. }
$$

Let $\epsilon>0$ be fixed. Then at almost all points the inequality

$$
s_{n} \phi_{n}<S_{n}<s_{n}\left(\phi_{n}+3 \epsilon / \phi_{n}\right)
$$

will be satisfied for infinitely many $n$.

Before proceeding with the proof let us remark that, putting

$$
p_{k}=\left(1 / \phi_{k}^{\prime}\right) \exp \left\{-(1 / 2) \phi_{k}{ }^{2}\left[1+Q_{k}^{\prime}\left(\phi_{k}^{\prime}\right)\right]\right\},
$$

condition (4.1) is equivalent to

$$
\sum p_{k} \in \mathcal{D}
$$

Indeed, by Theorem 1 of L.T.

$$
\operatorname{Pr}\left\{S_{k}^{\prime}>s_{k}^{\prime} \phi_{k}^{\prime}\right\}<\text { const. } p_{k} \text {. }
$$

Moreover, by Theorem 2 of that paper, to any $\epsilon>0$ there correspond two con- 
stants $0<a_{1}<a_{2}$, depending only on $\epsilon$, such that

$$
a_{1} p_{k}<\operatorname{Pr}\left\{s_{k}^{\prime} \phi_{k}^{\prime}<S_{k}^{\prime}<s_{k}^{\prime}\left(\phi_{k}^{\prime}+\epsilon / \phi_{k}^{\prime}\right)\right\}<a_{2} p_{k} .
$$

Accordingly, (4.1) is equivalent to the statement that

$$
\sum \operatorname{Pr}\left\{s_{k}^{\prime} \phi_{k}^{\prime}<S_{k}^{\prime}<s_{k}^{\prime}\left(\phi_{k}^{\prime}+\epsilon / \phi_{k}^{\prime}\right)\right\} \in \mathcal{D} \text {. }
$$

4.1. For the proof of Lemma 1 we require a sequence $\left\{k_{\nu}\right\}$ defined by the recurrence relations

$$
s_{k_{v+1}}^{\prime} \leqq M s_{k_{v}}^{\prime} \phi_{k_{v}}^{\prime}, \quad s_{k_{v+1+1}}^{\prime}>M s_{k_{v}}^{\prime} \phi_{k_{\nu}}^{\prime}
$$

where $M>1$ is a constant to be determined later; $k_{1}$ can be chosen arbitrarily. It is readily seen that

$$
(2 /(2+b)) M s_{k_{\nu}}^{\prime} \phi_{k_{\nu}}^{\prime}<s_{k_{\nu+1}}^{\prime} \leqq M s_{k_{\nu}}^{\prime} \phi_{k_{\nu}}^{\prime}
$$

and

$$
0<k_{v+1}-k_{\nu} \leqq\left(M^{2} / a\right) \phi_{k_{v+1}}^{\prime 4}
$$

In fact, using (4.7), (2.20), (3.4), and (2.2) it follows that

$$
M^{2} s_{k_{\nu}}^{\prime 2} \phi_{k_{\nu}}^{\prime 2}<s_{k_{\nu+1}+1}^{\prime 2} \leqq{s_{k_{\nu+1}}^{\prime 2}}^{\prime 2}\left(1+b / \phi_{k_{\nu+1}}^{\prime 2}\right) \leqq(1+b / 4) s_{k_{\nu+1}}^{\prime 2},
$$

which obviously implies (4.8). In the same way

$$
\begin{aligned}
\phi_{k_{\nu}}^{\prime 2} & \geqq \frac{1}{M^{2}} \frac{s_{k_{\nu+1}}^{\prime 2}}{s_{k_{\nu}}^{\prime 2}} \geqq \frac{1}{M^{2}} \cdot \prod_{n=k_{\nu}}^{k_{\nu+1}-1}\left(1+\frac{a}{\phi_{n}^{\prime 2}}\right) \geqq \frac{1}{M^{2}}\left(1+\frac{a}{\phi_{k_{\nu+1}}^{\prime 2}}\right)^{\left(k_{\nu+1}-k_{\nu}\right)} \\
& \geqq \frac{1}{M^{2}} \frac{a}{\phi_{k_{\nu+1}}^{\prime 2}}\left(k_{\nu+1}-k_{\nu}\right),
\end{aligned}
$$

which proves (4.9).

4.2. For any $i<j$ let us define

$$
S_{i, j}^{\prime}=S_{i}^{\prime}-S_{i}^{\prime}=\sum_{n=n_{i}+1}^{n_{j}} X_{n} .
$$

Then

$$
F_{i, j}^{\prime}(x)=\operatorname{Pr}\left\{S_{i, j}^{\prime} \leqq x\right\}
$$

is the distribution function of $S_{i, j}^{\prime}$, and obviously

$$
\int_{-\infty}^{+\infty} x d F_{i, i}^{\prime}(x)=0
$$

and 


$$
s_{i, j}^{\prime 2} \equiv \int_{-\infty}^{+\infty} x^{2} d F_{i, j}^{\prime}(x)=s_{i}^{\prime 2}-s_{i}^{\prime 2}
$$

Now denote by $A_{\nu, m}\left(m \geqq k_{\nu}\right)$ the set

$$
A_{v, m}=\underset{p}{E}\left\{s_{m}^{\prime}\left(\phi_{m}^{\prime}+\epsilon / \phi_{m}^{\prime}\right)<S_{k_{\nu-1}, m}^{\prime}<s_{m}^{\prime}\left(\phi_{m}^{\prime}+2 \epsilon / \phi_{m}^{\prime}\right)\right\},
$$

where $\epsilon$ is an arbitrary, but fixed, number with

$$
0<\epsilon<1 \text {. }
$$

We propose now to show: if

$$
\begin{aligned}
& k_{\nu} \leqq m<k_{\nu+1}, \\
& \phi_{k_{\nu+1}}^{\prime}<2 \phi_{k_{\nu-1}}^{\prime},
\end{aligned}
$$

and if

$$
(2+b)^{2} / M^{2}<\epsilon / 4,
$$

then there exists a constant $\eta>0$ (depending only on $\epsilon$ ) such that

$$
\operatorname{Pr}\left(A_{\nu, m}\right)>\eta p_{m}
$$

for all $\nu$ sufficiently large.

Proof. Since the sequence $s_{k}^{\prime}$ is not decreasing and $m>k$, we obtain, using (4.16), (4.8), (4.17), and (4.18),

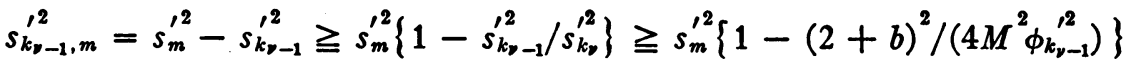

$$
\begin{aligned}
& \geqq s_{m}^{\prime 2}\left\{1-(2+b)^{2} /\left(M^{2} \phi_{m}^{\prime 2}\right)\right\} \geqq s_{m}^{\prime 2}\left\{1-\epsilon /\left(4 \phi_{m}^{\prime 2}\right)\right\} \text {, }
\end{aligned}
$$

whence

$$
s_{m}^{\prime}\left(1-\epsilon /\left(4 \phi_{m}^{\prime 2}\right)\right) \leqq \dot{s}_{k_{v-1}, m} \leqq \dot{s}_{m}
$$

Accordingly, putting

$$
A_{\nu, m}^{*}=\underset{p}{E}\left\{s_{k_{\nu-1}, m}^{\prime}\left(\phi_{m}^{\prime}+3 \epsilon /\left(2 \phi_{m}^{\prime}\right)\right) \leqq S_{k_{\nu-1}, m}^{\prime} \leqq s_{k_{\nu-1}, m}^{\prime}\left(\phi_{m}^{\prime}+2 \epsilon / \phi_{m}^{\prime}\right)\right\}
$$

it is readily seen that

$$
A_{\nu, m}^{*} \subset A_{\nu, m}
$$

To evaluate $\operatorname{Pr}\left(A_{v, m}^{*}\right)$ we can apply Theorems 1 and 2 of L.T. Indeed, $S_{k_{p-1}, m}^{\prime}$ is a sum of independent random variables $X_{k}$; moreover, it is easily seen (using (4.20), (4.15), (2.2) and (2.6)) that

$$
\text { l.u.b. }\left|X_{k}\right|<\lambda_{n_{m}} s_{m}^{\prime}(17 / 16) \lambda_{n_{m}} s_{k_{\nu-1}, m}^{\prime}<(1 / 100) s_{k_{\nu-1}, m}^{\prime},
$$


so that Theorem 2 is applicable. It follows immediately that

$$
\operatorname{Pr}\left(A_{v, m}\right) \geqq \operatorname{Pr}\left(A_{v, m}^{*}\right) \geqq \eta p_{m} .
$$

4.3. Now put

$$
A_{\nu}=\sum_{m=k_{\nu}}^{k_{\nu+1}-1} A_{\nu, m}
$$

and

$$
P_{\nu}=\sum_{m=k_{v}}^{k_{v+1}-1} p_{m}
$$

We shall show: If (4.17) and (4.19) hold, then there exists a constant $\eta_{1}>0$ such that

$$
\operatorname{Pr}\left(A_{v}\right)>\eta_{1} P_{v}
$$

for all $\nu$ sufficiently large.

Proof. Let $N$ be a fixed integer and consider the sets

$$
A_{\nu, m}^{(N)}=A_{\nu, m}-A_{\nu, m} \sum_{\rho=m+N}^{k_{\nu+1}-1} A_{\nu, \rho} .
$$

Obviously

$$
A_{\nu}=\sum_{m=k_{\nu}}^{k_{\nu+1}-1} A_{\nu, m}^{(N)} .
$$

On the other hand, it follows from (4.26) that the intersection of two sets $A_{\nu, m_{1}}^{(N)}$ and $A_{\nu, m_{2}}^{(N)}$ will be empty unless $\left|m_{1}-m_{2}\right|<N$. Any point of $A_{\nu}$ is therefore contained in at most $N$ distinct sets $A_{\nu, m}^{(N)}$ figuring in (4.27) so that

$$
\operatorname{Pr}\left(A_{\nu}\right) \geqq \frac{1}{N} \sum_{m=k_{\nu}}^{k_{v+1}-1} \operatorname{Pr}\left(A_{\nu, m}^{(N)}\right) .
$$

Again it follows from the definition (4.14) of $A_{r, m}$ that for any point of $A_{\nu, m} A_{\nu, \rho}(\rho>m)$

(4.29) $S_{m, \rho}^{\prime}>s_{\rho}^{\prime}\left(\phi_{\rho}^{\prime}+\epsilon / \phi_{\rho}^{\prime}\right)-s_{m}^{\prime}\left(\phi_{m}^{\prime}+2 \epsilon / \phi_{m}^{\prime}\right)>\left(s_{\rho}^{\prime}-s_{m}^{\prime}\right) \phi_{\rho}^{\prime}-s_{m}^{\prime}\left(2 \epsilon / \phi_{m}^{\prime}\right)$.

In other words

$$
A_{\nu, m} A_{\nu, p} \subset B_{m, p}
$$

where

$$
B_{m, p}=\underset{p}{E}\left\{S_{m, p}^{\prime}>\left(s_{p}^{\prime}-s_{m}^{\prime}\right) \phi_{\rho}^{\prime}-s_{m}^{\prime}\left(2 \epsilon / \phi_{m}^{\prime}\right)\right\}
$$


However, the sets $A_{v, m}$ and $B_{m, p}$ are independent (see the footnote 5 on p. 375), so that

$$
P\left(A_{v, m} B_{m, \rho}\right)=P\left(A_{v, m}\right) P\left(B_{m, \rho}\right) .
$$

Comparing (4.26), (4.28), (4.30), (4.32), and (4.19) we see that

$$
\begin{aligned}
\operatorname{Pr}\left(A_{\nu}\right) & \geqq \frac{1}{N} \sum_{m=k_{\nu}}^{k_{\nu+1}-1} \operatorname{Pr}\left\{A_{\nu, m}-\sum_{\rho=m+N}^{k_{\nu+1}-1} A_{\nu, m} B_{m, \rho}\right\} \\
& \geqq \frac{1}{N} \sum_{m=k_{\nu}}^{k_{\nu+1}-1} \operatorname{Pr}\left(A_{\nu, m}\right)\left\{1-\sum_{\rho=m+N}^{k_{\nu+1}-1} \operatorname{Pr}\left(B_{m, \rho}\right)\right\} \\
& \geqq \frac{\eta}{N} \sum_{m=k_{\nu}}^{k_{\nu+1}-1} \operatorname{pr}_{m}\left\{1-\sum_{\rho=m+N}^{k_{\nu+1}-1} \operatorname{Pr}\left(B_{m, \rho}\right)\right\} .
\end{aligned}
$$

We turn to the evaluation of $\operatorname{Pr}\left(B_{m, \rho}\right)$. For that purpose we write the inequality in (4.31) in the form

$$
S_{m, \rho}^{\prime}>s_{m, \rho}^{\prime} \phi_{\rho}^{*}
$$

where

$$
\phi_{\rho}^{*}=\frac{s_{\rho}^{\prime}-s_{m}^{\prime}}{s_{m, \rho}^{\prime}} \phi_{\rho}^{\prime}-\frac{s_{m}^{\prime}}{s_{m, \rho}^{\prime}} \cdot \frac{2 \epsilon}{\phi_{m}^{\prime}} .
$$

To evaluate the probability of (4.34) we may again apply Theorem 1 of L.T., provided that all the $X_{n}$ with $n<n_{\rho}$ have an upper bound not exceeding, say, $\left(1 / 200 \phi_{\rho}^{*}\right) s_{m, \rho}^{\prime}$. Now for $n<n_{\rho}$

$$
\left|X_{n}\right|<\lambda_{\rho}^{\prime} s_{\rho}^{\prime} \leqq \frac{1}{200 \phi_{\rho}^{\prime}} \frac{s_{p}^{\prime}}{s_{m, p}^{\prime}} s_{m, \rho}^{\prime}
$$

and by (4.35)

$$
\frac{\phi_{\rho}^{*}}{\phi_{\rho}^{\prime}} \frac{s_{\rho}^{\prime}}{s_{m, p}^{\prime}}<\frac{s_{\rho}^{\prime}\left(s_{\rho}^{\prime}-s_{m}^{\prime}\right)}{s_{m, \rho}^{\prime 2}}=\frac{s_{\rho}^{\prime}}{s_{m}^{\prime}+s_{\rho}^{\prime}}<1 .
$$

Theorem 1 of L.T. is therefore applicable and

$$
\begin{aligned}
\operatorname{Pr}\left(B_{m, \rho}\right) & =\operatorname{Pr}\left\{S_{m, \rho}^{\prime}>s_{m, \rho}^{\prime} \phi_{\rho}^{*}\right\} \\
& <\eta_{2} \frac{1}{\phi_{\rho}^{*}} \exp \left\{-(1 / 2) \phi_{\rho}^{*^{2}}\left[1+Q_{n_{\rho}-n_{m}}^{*}\left(\phi_{\rho}^{*}\right)\right]\right\}
\end{aligned}
$$

provided, say, $\phi_{\rho}^{*}>2$. Using the estimates (2.4) for the coefficients of $Q^{*}$ it is readily seen that

$$
\operatorname{Pr}\left(B_{m, \rho}\right) \leqq \eta_{2} e^{(-2 / 5) \phi_{\rho}^{* 2}}
$$


Now by (4.35)

$$
\begin{aligned}
\phi_{\rho}^{*} & =\frac{s_{\rho}^{\prime}-s_{m}^{\prime}}{\left(s_{\rho}^{\prime 2}-s_{m}^{\prime 2}\right)^{1 / 2}} \phi_{\rho}^{\prime}-\frac{s_{m}^{\prime}}{\left(s_{\rho}^{\prime 2}-s_{m}^{\prime 2}\right)^{1 / 2}} \frac{2 \epsilon}{\phi_{m}^{\prime}} \\
& =\frac{\left(s_{\rho}^{\prime 2}-s_{m}^{\prime 2}\right)^{1 / 2}}{s_{\rho}^{\prime}+s_{m}^{\prime}} \phi_{\rho}^{\prime}-\frac{s_{m}^{\prime}}{\left(s_{\rho}^{\prime 2}-s_{m}^{\prime 2}\right)^{1 / 2}} \frac{2 \epsilon}{\phi_{m}^{\prime}} \\
& \geqq \frac{1}{2}\left(1-\frac{s_{m}^{\prime 2}}{s_{\rho}^{\prime 2}}\right)^{1 / 2} \phi_{\rho}^{\prime}-\frac{1}{\left(s_{\rho}^{\prime 2} / s_{m}^{\prime 2}-1\right)^{1 / 2}} \frac{2 \epsilon}{\phi_{m}^{\prime}} .
\end{aligned}
$$

However, by means of (2.20), (3.4), (4.16) and (4.17) we deduce that for $\rho<k_{v+1}$

$$
\left(\frac{s_{\rho}^{\prime}}{s_{m}^{\prime}}\right)^{2} \geqq \prod_{n=m}^{\rho-1}\left(1+\frac{a}{\phi_{n}^{\prime 2}}\right) \geqq\left(1+\frac{a}{4 \phi_{m}^{\prime 2}}\right)^{\rho-m} \geqq 1+\frac{a(\rho-m)}{4 \phi_{m}^{\prime 2}} .
$$

This inequality will be applied in three ways.

(i) Since $\rho \geqq m+N$ it follows that

$$
s_{\rho}^{\prime 2} / s_{m}^{\prime 2} \geqq 1+a N /\left(4 \phi_{\rho}^{\prime 2}\right) ;
$$

using (4.17) it is therefore seen that the last term of the right-hand member in (4.38) is small if $N$ is large. Hence (4.38) implies, for $N$ sufficiently large,

$$
\phi_{\rho}^{*} \geqq(1 / 2)\left(1-s_{m}^{\prime 2} / s_{\rho}^{\prime 2}\right)^{1 / 2} \phi_{\rho}^{\prime}-a^{1 / 2} / 10 \text {. }
$$

(ii) Consider now $\rho$ with

$$
a(\rho-m) \leqq \phi_{m}^{\prime 2}
$$

Combining (4.39)-(4.41) we obtain

$$
\phi_{\rho}^{*} \geqq \frac{1}{2}\left\{1-\frac{1}{1+a(\rho-m) /\left(4 \phi_{m}^{\prime 2}\right)}\right\}^{1 / 2} \phi_{\rho}^{\prime}-\frac{a^{1 / 2}}{10} \geqq \frac{a^{1 / 2}}{10}(\rho-m)^{1 / 2} .
$$

Comparing (4.37) with (4.42) it is readily seen that, for $N$ sufficiently large,

$$
\sum_{\rho=m+N}^{\min \left\{k_{\nu+1}-1, m+\left[a-1 \phi_{m}^{\prime 2}\right]\right\}} \operatorname{Pr}\left(B_{m, \rho}\right) \leqq \eta_{2} \sum_{\rho=m+N}^{\infty} e^{-(a / 250)(\rho-m)}<\frac{1}{3} .
$$

(iii) Finally, consider $\rho$ with

$$
a(\rho-m) \geqq \phi_{m}^{\prime 2}
$$

For these we obtain from (4.39) $s_{\rho}^{\prime 2} / s_{m}^{\prime 2} \geqq 5 / 4$ and from (4.40)

$$
\phi_{\rho}^{*} \geqq(1 / 10)\left(\phi_{\rho}^{\prime}-a^{1 / 2}\right) ;
$$


since $k_{\nu}<\rho<k_{\nu+1}$ and since $\phi_{n}^{\prime} \rightarrow \infty$, it follows from (4.45) and (4.17) that for $\nu$ sufficiently large

$$
\phi_{\rho}^{*} \geqq(1 / 30) \phi_{k_{\nu+1}}^{\prime}
$$

Combining (4.37) and (4.46) shows that

$$
\begin{aligned}
\sum_{\rho=m+\left[\phi_{m}^{\prime} / a\right]}^{k_{\nu+1}-1} \operatorname{Pr}\left(B_{m, \rho}\right) & <\eta_{2} \sum_{\rho=m+\left[\phi_{m}^{\prime 2} / a\right]}^{k_{\nu+1}-1} e^{-(1 / 2500) \phi_{k_{\nu+1}^{\prime}}} \\
& \leqq \eta_{2}\left(k_{\nu+1}-k_{\nu}\right) e^{-(1 / 2500) \phi_{k_{\nu+1}^{\prime}}^{\prime}}
\end{aligned}
$$

However, (4.9) implies that the last expression tends to zero as $\nu \rightarrow \infty$. Accordingly, for $\nu$ sufficiently large,

$$
\sum_{\rho=m+\left[\phi_{m}^{\prime 2} / a\right]}^{k \nu+1-1} \operatorname{Pr}\left(B_{m, \rho}\right)<\frac{1}{3} .
$$

Substituting for $\operatorname{Pr}\left(B_{m, p}\right)$ from (4.43) and (4.48) in (4.33) we obtain

$$
\operatorname{Pr}\left(A_{v}\right) \geqq \frac{\eta}{3 N} \sum_{m=k_{\nu}}^{k v+1-1} p_{m}=\frac{\eta}{3 N} P_{\nu}
$$

which proves the assertion (4.25).

4.4. We propose now to show that almost all points belong to infinitely many $A$, or, in other words, that

$$
\operatorname{Pr}\left(\sum_{\nu=\bar{\nu}}^{\infty} A_{\nu}\right)=1
$$

for any $\bar{\nu}$. For that purpose we note that the set $A$, depends only on the variables $X_{n}$ with $n_{k_{v-1}} \leqq n<n_{k_{\nu+1}}$ (cf. (4.14) and (4.23)). Accordingly, the sets $A_{2}$, are mutually independent, and

$$
\operatorname{Pr}\left(\sum_{\nu=\bar{\nu}}^{\infty} A_{\nu}\right) \geqq \operatorname{Pr}\left(\sum_{\nu=\bar{\nu}}^{\infty} A_{2 \nu}\right)=1-\prod_{\nu=\bar{\nu}}^{\infty}\left(1-\operatorname{Pr}\left(A_{2 \nu}\right)\right),
$$

and a similar relationship holds for odd subscripts. Now it would be trivial to deduce (4.49) from (4.50), if we could use (4.25). However, the last inequality has been proved only under the assumption that (4.17) holds. We shall show that the $\nu$ 's for which (4.17) does not hold are of no influence.

Let $\left.{ }^{15}\right)\{\nu(r)\}, r=1, \cdots$, denote those integers for which

$$
\phi_{k_{\nu,+1}}^{\prime} \geqq 2 \phi_{k_{\boldsymbol{\nu}(r)-1}^{\prime}}^{\prime}
$$

We shall prove that

$$
\sum_{r} P_{\nu(r)} \in \mathcal{C}
$$

(15) For simplicity in print the notation $\nu(r)$ has been introduced instead of the usual $\nu_{r}$. 
In fact, it follows readily from $(2.6),(2.4)$ and $(4.3)$ that $p_{k} \leqq\left(1 / \phi_{k}^{\prime}\right) e^{(-2 / 5) \phi_{k}^{\prime 2}}$, and therefore

$$
p_{k} \leqq\left(1 / \phi_{k}^{\prime 4}\right) e^{-\phi_{k}^{\prime}}
$$

for $k$ sufficiently large. We find by means of (4.8) and (2.20)

$$
\phi_{k_{\nu}}^{\prime 2} \geqq \frac{1}{M^{2}} \frac{s_{k_{\nu+1}}^{\prime 2}}{s_{k_{\nu}}^{\prime 2}} \geqq \frac{1}{M^{2}} \prod_{m=k_{\nu}}^{k_{\nu+1}-1}\left(1+\frac{a}{\phi_{m}^{\prime 2}}\right) \geqq \frac{a}{M^{2}} \sum_{m=k_{\nu}}^{k_{\nu+1}-1} \frac{1}{\phi_{m}^{\prime 2}} .
$$

Thus by (4.53) and (2.2)

or

$$
P_{\nu}=\sum_{m=k_{\nu}}^{k_{\nu+1-1}} p_{m} \leqq \frac{e^{-\phi_{k_{\nu}}^{\prime}}}{\phi_{k_{\nu}}^{\prime 2}} \sum_{m=k_{\nu}}^{k_{\nu+1}-1} \frac{1}{\phi_{m}^{\prime 2}} \leqq \frac{M^{2}}{a} e^{-\phi_{k_{\nu}}^{\prime}}
$$

$$
\sum P_{\nu(r)} \leqq \frac{M^{2}}{a} \sum_{r} e^{-\phi^{\prime} k_{\nu(r)}}
$$

but (4.51) implies that the sequence $\left\{\phi_{k_{(r)}}^{\prime}\right\}$ increases so rapidly that the series (4.54) converges. Accordingly the series $\sum_{\nu \neq \nu(r)} P_{\nu}$ diverges, and without loss of generality we may suppose that $\sum_{2 v \neq v(r)} P_{2}$, diverges. It follows from the definition (4.51) that for $\nu \neq \nu(r),(4.17)$ holds. Accordingly, for these $\nu$ the inequality (4.25) holds, and therefore

$$
\begin{aligned}
\operatorname{Pr}\left(\sum_{\nu \geqq \bar{\nu}, 2 v \neq \nu(r)} A_{2 v}\right) & =1-\prod_{\nu \geqq \bar{\nu}, 2 v \neq \nu(r)}\left(1-\operatorname{Pr}\left(A_{2 v}\right)\right) \\
& \geqq 1-\prod_{\nu \geq \bar{\nu}, 2 v \neq \nu(r)}\left(1-\eta_{1} P_{\nu}\right)=1 .
\end{aligned}
$$

4.5. The proof of Lemma 1 is now easily accomplished. We know that almost all points belong to infinitely many sets $A_{2}$, with $2 \nu \neq \nu(r)$, that is to say, such that

$$
\phi_{k_{2 \nu+1}}^{\prime}<2 \phi_{k_{2 \nu-1}}^{\prime}
$$

Denote then by $C_{2}$, the set

$$
C_{2 \nu}=\underset{p}{E}\left\{-(1 / \delta) s_{k_{2 \nu-1}}^{\prime}<S_{k_{2 \nu-1}}^{\prime}<(1 / \delta) s_{k_{2 \nu+1}}^{\prime}\right\},
$$

where $\delta>0$ is arbitrary. At any point of the intersection $A_{2 v, m} C_{2 \nu}$ we have simultaneously the inequality figuring in (4.57) and

$$
s_{m}^{\prime}\left(\phi_{m}^{\prime}+\epsilon / \phi_{m}^{\prime}\right)<S_{k_{2 v-1}, m}^{\prime}<s_{m}^{\prime}\left(\phi_{m}^{\prime}+2 \epsilon / \phi_{m}^{\prime}\right) .
$$

Adding these inequalities we find that in $A_{2 v, m} C_{2}$, (4.59) $s_{m}^{\prime} \phi_{m}^{\prime}+s_{m}^{\prime} \epsilon / \phi_{m}^{\prime}-(1 / \delta) s_{k_{2 v-1}}^{\prime}<S_{m}{ }^{\prime}<s_{m}{ }^{\prime} \phi_{m}{ }^{\prime}+2 \epsilon s_{m}^{\prime} / \phi_{m}^{\prime}+(1 / \delta) s_{k_{2 v+1}}^{\prime}$. 
However, for $k_{2 v} \leqq m<k_{2 v+1}$ we have, using (4.8) and (4.56),

$$
\frac{s_{k_{2 v-1}}^{\prime}}{s_{m}^{\prime}} \leqq \frac{s_{k_{2 v-1}}^{\prime}}{s_{k_{2 v}}^{\prime}} \leqq \frac{2+b}{2 M} \frac{1}{\phi_{k_{2 v-1}}^{\prime}} \leqq \frac{2+b}{M} \frac{1}{\phi_{m}^{\prime}} ;
$$

thus, if the constant $M$ is chosen so that

$$
(2+b) / M<\epsilon \delta
$$

we obtain from (4.59) that at any point of $A_{2 v, m} C_{2 v}$

$$
s_{m}^{\prime} \phi_{m}^{\prime}<S_{m}^{\prime}<s_{m}^{\prime}\left(\phi_{m}^{\prime}+3 \epsilon / \phi_{m}^{\prime}\right) \text {. }
$$

Denote the set of points for which (4.61) holds by $\Lambda_{m}$. Then $\Lambda_{m} \supset A_{2 v, m} C_{2 \text { v }}$, and therefore (always for $2 \nu \neq \nu(r)$ )

$$
\operatorname{Pr}\left(\sum_{m=k_{2 v}}^{k_{2 v+1}-1} \Lambda_{m}\right) \geqq \operatorname{Pr}\left(A_{2 \nu} C_{2 v}\right)
$$

or

$$
\operatorname{Pr}\left(\sum_{m \geqq k_{\bar{\nu}}} \Lambda_{m}\right) \geqq \operatorname{Pr}\left(\sum_{\nu \geqq \bar{\nu}, 2 \nu \neq \nu(r)} A_{2 \nu} C_{2 \nu}\right) .
$$

Again, it follows from the definition (4.57) that (Tschebycheff's inequality)

$$
\begin{aligned}
\operatorname{Pr}\left(C_{2 v}\right) & =\int_{|x|<(1 / \delta) s_{k_{2 \nu-1}}^{\prime}} d F_{k_{2 \nu-1}}^{\prime}=1-\int_{|x| \geqq(1 / \delta) s_{k_{2 \nu-1}}^{\prime}} d F_{k_{2 \nu-1}}^{\prime} \\
& \geqq 1-\left(\delta^{2} / s_{k_{2 \nu-1}}^{\prime 2}\right) \int_{|x| \geqq(1 / \delta) s_{2 \nu-1}^{\prime}} x^{2} d F_{k_{2 \nu-1}}^{\prime} \geqq 1-\delta^{2} .
\end{aligned}
$$

On the other hand, the sets $A_{2 \nu}$ and $C_{2 \rho}$ are independent if $\rho \geqq \nu$ (cf. footnote 5 on p. 375$)$. We have therefore

$$
\begin{aligned}
\operatorname{Pr}\left(\sum_{\nu \geqq \bar{\nu}, 2 \nu \neq \nu(r)} A_{2 \nu} C_{2 \nu}\right) & =\sum_{\nu \geqq \bar{\nu}, 2 \nu \neq \nu(r)} \operatorname{Pr}\left\{A_{2 \nu} C_{2 \nu}-A_{2 \nu} C_{2 \nu} \sum_{\rho>\nu, 2 \rho \neq \nu(r)} A_{2 \rho} C_{2 \rho}\right\} \\
& \geqq \sum_{\nu \geqq \bar{\nu}, 2 \nu \neq \nu(r)} \operatorname{Pr}\left\{C_{2 \nu}\left(A_{2 \nu}-A_{2 \nu} \sum_{\rho>\nu, 2 \rho \neq \nu(r)} A_{2 \rho}\right)\right\} \\
& =\sum_{\nu \geqq \bar{\nu}, 2 \nu \neq \nu(r)} \operatorname{Pr}\left(C_{2 \nu}\right) \operatorname{Pr}\left(A_{2 \nu}-A_{2 \nu} \sum_{\rho>\nu, 2 \rho \neq \nu(r)} A_{2 \rho}\right) \\
& \geqq\left(1-\delta^{2}\right) \operatorname{Pr}\left(\sum_{\nu \geqq \bar{\nu}, 2 \nu \neq \nu(r)} A_{2 \nu}\right)=\left(1-\delta^{2}\right)
\end{aligned}
$$

(by (4.55)). This proves that all points, with the possible exception of a set of measure $\delta^{2}$, belong to infinitely many sets $\Lambda_{m}$; at such points the inequality 
(4.61) is satisfied for infinitely many values $m$. Since $\delta$ is arbitrary, and the definition of $\Lambda_{m}$ is independent of $\delta$, it follows that at almost all points the inequality (4.61) will be satisfied for infinitely many $m$. This proves Lemma 1.

5. Lemma 2. Suppose that condition (2.6) is satisfied and that

$$
\sum \operatorname{Pr}\left\{S_{k}^{\prime}>s_{k}^{\prime} \phi_{k}^{\prime}\right\} \in \mathcal{C} \text {. }
$$

Then

$$
\left\{\phi_{n}\right\} \in \mathcal{L} \text {. }
$$

As before (cf. $\$ 4$ ) we see that condition (5.1) is equivalent to

$$
\sum p_{k} \in \mathcal{C}
$$

where $p_{k}$ is defined by (4.3). We shall require three sets:

$$
\begin{aligned}
A_{n} & =\underset{p}{E}\left\{S_{n}>s_{n} \phi_{n}\right\}, \\
B_{n, k} & =\underset{p}{E}\left\{S_{n, n_{k}}>-2 s_{n, n_{k}}\right\}, \\
C_{k} & =\underset{p}{E}\left\{S_{n_{k}}>s_{n_{k}}\left(\phi_{k-1}^{\prime}-(2+2 b) / \phi_{k-1}^{\prime}\right)\right\}
\end{aligned}
$$

(for the definition of $S_{n, m}$ and $s_{n, m}$ cf. (4.10) and (4.13)). It is sufficient to prove that

$$
P\left(\sum_{n=N}^{\infty} A_{n}\right) \leqq \epsilon
$$

for $N$ sufficiently large.

Again we deduce from (2.20) for $n_{k-1}<n \leqq n_{k}$

$$
s_{n}^{2} \geqq s_{n_{k-1}}^{2} \geqq s_{n_{k}}^{2} /\left(1+b / \phi_{n_{k-1}}^{2}\right) \geqq s_{n_{k}}^{2}\left(1-b / \phi_{n_{k-1}}^{2}\right) ;
$$

on the other hand by definition and by (5.8)

$$
s_{n, n_{k}}^{2}=s_{n_{k}}^{2}-s_{n}^{2} \leqq\left(b / \phi_{n_{k-1}}^{2}\right) s_{n_{k}}^{2} .
$$

Adding the inequalities in (5.4) and (5.5) and using again (5.8) we see therefore that at all points of the intersection $A_{n} B_{n, k}$

$$
\begin{aligned}
S_{n_{k}} \equiv S_{n}+S_{n, n_{k}} & >s_{n_{k}}\left(\phi_{n}-b \phi_{n} / \phi_{n_{k-1}}^{2}-2 b^{1 / 2} / \phi_{n_{k-1}}\right) \\
=s_{n_{k}}\left(\phi_{n_{k-1}}+\right. & {\left.\left[\phi_{n}-\phi_{n_{k-1}}\right]\left[1-b / \phi_{n_{k-1}}\right]-\left[b+2 b^{1 / 2}\right] / \phi_{n_{k-1}}\right) } \\
& >s_{n_{k}}\left(\phi_{n_{k-1}}-(2+2 b) / \phi_{n_{k-1}}\right),
\end{aligned}
$$

provided $\phi_{n_{k-1}}^{2}>b$. Comparing (5.6) with (5.9) it is seen that

$$
C_{k} \supset A_{n} B_{n, k},
$$$$
n_{k-1}<n \leqq n_{k} .
$$ 
Furthermore, using Tschebycheff's inequality (cf. (4.63)), we obtain

$$
\operatorname{Pr}\left(B_{n, k}\right) \geqq 3 / 4 \text {. }
$$

Now it should be noticed that the sets $B_{n, k}$ and $A_{m}$ are independent if $m \leqq n$. Therefore

$$
\begin{aligned}
\operatorname{Pr}\left(C_{k}\right) & \geqq \operatorname{Pr}\left(\sum_{n=n_{k-1}+1}^{n_{k}} A_{n} B_{n, k}\right) \\
& \equiv \sum_{n=n_{k-1}+1}^{n_{k}} \operatorname{Pr}\left\{A_{n} B_{n, k}-A_{n} B_{n, k} \sum_{m=n_{k-1+1}}^{n} A_{m} B_{m, k}\right\} \\
& \geqq \sum_{n=n_{k-1}+1}^{n_{k}} \operatorname{Pr}\left\{A_{n} B_{n, k}-A_{n} B_{n, k} \sum_{m=n_{k-1+1}}^{n} A_{m}\right\} \\
& =\sum_{n=n_{k-1}+1}^{n_{k}} \operatorname{Pr}\left(B_{n, k}\right) \operatorname{Pr}\left(A_{n}-A_{n} \sum_{m=n_{k-1}+1}^{n} A_{m}\right) \\
& \geqq \frac{3}{4} \operatorname{Pr}\left(\sum_{n=n_{k-1}+1}^{n_{k}} A_{n}\right)
\end{aligned}
$$

or

(5)

$$
\operatorname{Pr}\left(\sum_{n=n_{k-1}+1}^{n_{k}} A_{n}\right) \leqq \frac{4}{3} \operatorname{Pr}\left(C_{k}\right) .
$$

However, Theorem 2 of L.T. asserts that $\operatorname{Pr}\left(C_{k}\right)<\eta_{2} p_{k-1}$, where $\eta_{2}$ is a constant independent of $k$. Accordingly

$$
\operatorname{Pr}\left(\sum_{n=n_{N}}^{\infty} A_{n}\right) \leqq \sum_{k=N-1}^{\infty} \operatorname{Pr}\left(\sum_{n=n_{k-1}+1}^{n_{k}} A_{n}\right) \leqq \frac{4}{3} \eta_{2} \sum_{k=N-1}^{\infty} p_{k-1},
$$

which proves (5.7).

6. Lemma 3. Suppose that condition (2.6) is satisfied, and that the series (2.7) diverges (converges). Then

$$
\sum_{k} \frac{1}{\phi_{n_{k}}} e^{-(1 / 2) \phi_{n_{k}}^{2}\left(1+Q_{n_{k}}\left(\phi_{n_{k}}\right)\right)}
$$

diverges (converges) for any sequence $\left\{n_{k}\right\}$ satisfying (2.20).

REMARK. It was pointed out earlier (cf. the introductory remarks to $\S \S 4$ and 5 ) that (6.1) converges or diverges simultaneously with

$$
\sum_{k} \operatorname{Pr}\left\{S_{n_{k}}>s_{n_{k}} \phi_{n_{k}}\right\} \text {. }
$$


Thus Lemmas 1 and 2 together are equivalent to Theorem 9 with the sharper form for upper functions provided by Theorem 8 . The three lemmas together prove Theorem 1 and Theorem 8 . From the previous lemmas it follows that the behavior of (6.1) is necessarily the same for all sequences $\left\{n_{k}\right\}$ satisfying (2.20). However, it should be noted that the following proof is absolutely independent of the previously established results.

Proof. As before, let $\left\{n_{k}\right\}$ stand for an arbitrary but fixed sequence satisfying (2.20). Since $\sigma_{n}^{2} \leqq \lambda_{n}^{2} s_{n}^{2}=o\left(s_{n}^{2}\right)$, we shall have, for sufficiently large $\left({ }^{16}\right) n$

$$
2 \sigma_{n}^{2} / s_{n}^{2} \geqq \log s_{n}^{2} / s_{n-1}^{2}=-\log \left(1-\sigma_{n}^{2} / s_{n}^{2}\right) \geqq \sigma_{n}^{2} / s_{n}^{2},
$$

and hence

$$
\sum_{n=n_{k}+1}^{n_{k+1}} \sigma_{n}^{2} / s_{n}^{2} \leqq \log s_{n_{k+1}}^{2} / s_{n_{k}}^{2} \leqq \log \left(1+b / \phi_{n_{k}}^{2}\right) \leqq b / \phi_{n_{k}}^{2},
$$

and similarly

$$
\sum_{n=n_{k}+1}^{n k+1} \sigma_{n}^{2} / s_{n}^{2} \geqq(1 / 2) \log \left(1+a / \phi_{n_{k}}^{2}\right) \geqq a /\left(4 \phi_{n_{k}}^{2}\right) .
$$

Suppose that the series (2.7) diverges, and define the sequence $\left\{m_{k}\right\}$ by

$$
\begin{aligned}
\phi_{m_{k}} \exp \left\{-(1 / 2) \phi_{m_{k}}[1+\right. & \left.\left.Q_{m_{k}}\left(\phi_{m_{k}}\right)\right]\right\} \\
& =\max _{n_{k}<n \leqq n_{k+1}} \phi_{n} \exp \left\{-(1 / 2) \phi_{n}^{2}\left[1+Q_{n}\left(\phi_{n}\right)\right]\right\} .
\end{aligned}
$$

Then

$$
n_{k}<m_{k} \leqq n_{k+1}
$$

and by (6.4)

$$
\begin{aligned}
\sum_{n} \frac{\sigma_{n}^{2}}{s_{n}^{2}} \phi_{n} e^{-(1 / 2) \phi_{n}^{2}\left(1+Q_{n}\left(\phi_{n}\right)\right)} & \leqq \sum_{k} \phi_{m_{k}} e^{-(1 / 2) \phi_{m_{k}}^{2}\left(1+Q_{m_{k}}\left(\phi_{m_{k}}\right)\right)} \sum_{n=n_{k}+1}^{n_{k+1}} \frac{\sigma_{n}^{2}}{s_{n}^{2}} \\
& \leqq b \sum_{k} \frac{\phi_{m_{k}}}{\phi_{n_{k}}^{2}} e^{-(1 / 2) \phi_{m_{k}}^{2}\left(1+Q_{m_{k}}\left(\phi_{m_{k}}\right)\right)}
\end{aligned}
$$

Unfortunately, the ratio $\phi_{m_{k}} / \phi_{n_{k}}$ is not necessarily bounded. Still, we shall show that those $k$, for which $\phi_{m_{k}} / \phi_{n_{k}}$ is not near unity, can be discarded. For that purpose, we make the following simple remark, which will be useful in the sequel: If for a sequence $\left({ }^{15}\right) k(1)<k(2)<k(3)<\ldots$

$$
\phi_{n_{k(r)+1}}>\phi_{n_{k(r)}}+2 / \phi_{n_{k(r)}}
$$

\section{then}

(16) The argument leading from (6.3) to (6.4) and (6.5) is due to Cesàro; cf. Knopp, loc. cit. p. 292. 


$$
\sum_{r} e^{-(1 / 4) \phi_{n_{k(r)}^{2}}^{2}}
$$

converges. In fact, the terms of (6.10) decrease geometrically since $(1 / 4) \phi_{n_{k(r+1)}}^{2}$ $\geqq(1 / 4) \phi_{n_{k(r)}}^{2}+1$. Now it follows from (2.6) and (6.7) that, at least for $k$ sufficiently large,

$$
\phi_{m_{k}} e^{-(1 / 2) \phi_{m_{k}}^{2}\left(1+Q_{m_{k}}\left(\phi_{m_{k}}\right)\right)}>e^{-(1 / 4) \phi_{m_{k}}^{2}} \geqq e^{-(1 / 4) \phi_{n_{k}}^{2}},
$$

so that by (6.10)

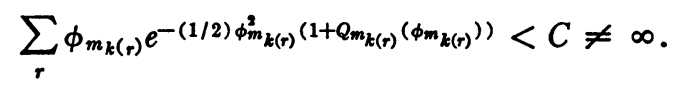

Again, if $k \neq k(1), k(2), \cdots$ we have

$$
\phi_{m_{k}} \leqq \phi_{n_{k+1}}<2 \phi_{n_{k}} .
$$

Using (6.12) and (6.13) we have

$\sum_{k} \frac{\phi_{m_{k}}}{\phi_{n_{k}}^{2}} e^{-(1 / 2) \phi_{m_{k}}^{2}\left(1+Q_{m_{k}}\left(\phi_{m_{k}}\right)\right)}=\sum_{k \neq k(r)}+\sum_{k(r)} \leqq 4 \sum_{k \neq k(r)} \frac{1}{\phi_{m_{k}}} e^{-(1 / 2) \phi_{m_{k}}^{2}\left(1+Q_{m_{k}}\left(\phi_{m_{k}}\right)\right)}+C ;$ therefore it follows from (6.8) that

$$
\sum_{k \neq k(r)} \frac{1}{\phi_{m_{k}}} e^{-(1 / 2) \phi_{m_{k}}^{2}\left(1+Q_{m_{k}}\left(\phi_{m_{k}}\right)\right)}
$$

diverges.

It will be noticed that the sequence $\left\{m_{k}\right\}$ does not necessarily satisfy the condition (2.20). We shall show that the divergence of (6.14) implies that of (6.1). It suffices obviously to show that if $k \neq k(1), k(2), \ldots$

$$
\frac{1}{\phi_{m_{k}}} e^{-(1 / 2) \phi_{m_{k}}^{2}\left(1+Q_{m_{k}}\left(\phi_{m_{k}}\right)\right)} \leqq C \frac{1}{\phi_{n_{k}}} e^{-(1 / 2) \phi_{n_{k}}^{2}\left(1+Q_{n_{k}}\left(\phi_{n_{k}}\right)\right)},
$$

where $C$ is a constant. Now $\phi_{m_{k}} \geqq \phi_{n_{k}}$. As for the exponential terms we have

$$
\begin{gathered}
\left|\phi_{m_{k}}^{2}\left(1+Q_{m_{k}}\left(\phi_{m_{k}}\right)\right)-\phi_{n_{k}}^{2}\left(1+Q_{n_{k}}\left(\phi_{n_{k}}\right)\right)\right| \leqq\left(\phi_{m_{k}}^{2}-\phi_{n_{k}}^{2}\right)\left(1+\left|Q_{n_{k}}\left(\phi_{n_{k}}\right)\right|\right) \\
+\phi_{m_{k}}^{2}\left|Q_{m_{k}}\left(\phi_{n_{k}}\right)-Q_{n_{k}}\left(\phi_{n_{k}}\right)\right|+\phi_{m_{k}}^{2}\left|Q_{m_{k}}\left(\phi_{m_{k}}\right)-Q_{m_{k}}\left(\phi_{n_{k}}\right)\right|
\end{gathered}
$$

Now $\left|Q_{n}\left(\phi_{n}\right)\right|<1 / 10$ by (2.6) and (2.4). Moreover, since $k \neq k(1), k(2), \cdots$, we have (cf. (6.9))

$$
\phi_{m_{k}}^{2}-\phi_{n_{k}}^{2} \leqq \phi_{n_{k+1}}^{2}-\phi_{n_{k}}^{2} \leqq 4+4 / \phi_{n_{k}}^{2}<5 .
$$

Thus the first term in (6.16) is less than 6.

Next, using Theorem 1, formula (2.8), of L.T. and (2.20) we find

$$
\stackrel{\phi_{m_{k}}^{2}}{2} \mid Q_{m_{k}}\left(\phi_{n_{k}}-Q_{n_{k}}\left(\phi_{n_{k}}\right) \mid \leqq 2 \phi_{n_{k}}^{2}\left(s_{m_{k}}^{2}-s_{n_{k}}^{2}\right) / s_{m_{k}}^{2} \leqq 2 \phi_{n_{k}}^{2}\left(s_{n_{k+1}}^{2}-s_{n_{k}}^{2}\right) / s_{n_{k}}^{2} \leqq 2 b,\right.
$$


so that also the second term in (6.16) is bounded. To appraise the last term we note that the derivative of $Q_{n}(x)$ is of the order of magnitude of $\lambda_{n}$. Thus, using the mean-value theorem we obtain by means of (2.6), (2.4), and (6.17)

$$
\phi_{m_{k}}^{2}\left|Q_{m_{k}}\left(\phi_{m_{k}}\right)-Q_{m_{k}}\left(\phi_{n_{k}}\right)\right|<2 \phi_{m_{k}}^{2} \lambda_{m_{k}}\left(\phi_{m_{k}}-\phi_{n_{k}}\right)<1,
$$

which proves (6.15) and, accordingly, the divergence of (6.1).

The same argument applies in the case of convergence.

7. Proof of Theorem 6. By virtue of Theorem 2 it suffices to prove: If $\phi(t)$ is a non-decreasing function, $\phi(t)>2$, then the integral (2.15) converges if, and only if,

$$
\sum \frac{\sigma_{n}^{2}}{s_{n}^{2}} \phi\left(s_{n}^{2}\right) e^{-(1 / 2) \phi^{2}\left(s_{n}^{2}\right)}
$$

converges. Since $(1 / t) \phi(t) e^{-(1 / 2) \phi^{2}(t)}$ is nonincreasing we have

$$
\begin{aligned}
\int_{s_{k}^{2}}^{\infty} \frac{\phi(t)}{t} e^{-(1 / 2) \phi^{2}(t)} d t & =\sum_{n=k+1}^{\infty} \int_{s_{n-1}^{2}}^{s_{n}^{2}} \frac{\phi(t)}{t} e^{-(1 / 2) \phi^{2}(t)} d t \\
& \geqq \sum_{n=k+1}^{\infty} \frac{s_{n}^{2}-s_{n-1}^{2}}{s_{n}^{2}} \phi\left(s_{n}^{2}\right) e^{-(1 / 2) \phi^{2}\left(s_{n}^{2}\right)},
\end{aligned}
$$

so that the convergence of (2.15) implies the convergence of (7.1). Similarly, if (2.15) diverges so does

$$
\sum \frac{\sigma_{n}^{2}}{s_{n-1}^{2}} \phi\left(s_{n-1}^{2}\right) e^{-(1 / 2) \phi^{2}\left(s_{n-1}^{2}\right)} .
$$

We have to show that the divergence of (7.2) implies that of (7.1). First let us observe that

$$
s_{n}^{2}=s_{n-1}^{2}+\sigma_{n}^{2}<s_{n-1}^{2}+\left(1 / 10^{4}\right) s_{n}^{2}
$$

or

$$
s_{n-1}^{2}>(1 / 2) s_{n}^{2}
$$

Furthermore, let $\nu_{k}$ denote those integers for which

$$
\phi\left(s_{v_{k}}^{2}\right)>\phi\left(s_{v_{k}-1}^{2}\right)+1 / \phi\left(s_{v_{k}-1}^{2}\right) .
$$

Since $\nu_{k-1} \leqq \nu_{k}-1$ this implies that $\phi^{2}\left(s_{\nu_{k}}^{2}\right)>\phi^{2}\left(s_{0}\right)+2 k$, which, together with (7.3), shows that

$$
\sum_{k} \frac{\sigma_{\nu_{k}}^{2}}{s_{\nu_{k}-1}^{2}} \phi\left(s_{\nu_{k}-1}^{2}\right) e^{-(1 / 2) \phi^{2}\left(s_{\nu_{k}-1}^{2}\right)} \in \mathcal{C}
$$


Thus, by assumption, the partial sum in (7.2) extended over $n \neq \nu_{1}, \nu_{2}, \cdots$, diverges; however, for these $n$

$$
\left(\sigma_{n}^{2} / s_{n-1}^{2}\right) \phi\left(s_{n-1}^{2}\right) e^{-(1 / 2) \phi^{2}\left(s_{n-1}^{2}\right)}<2 e^{3}\left(\sigma_{n}^{2} / s_{n}^{2}\right) \phi\left(s_{n}^{2}\right) e^{-(1 / 2) \phi^{2}\left(s_{n}^{2}\right)},
$$

which completes the proof.

8. Proof of Theorem 11. Writing, for abbreviation,

$$
\eta_{n}=2\left(\log \log s_{n}\right)^{1 / 2}
$$

we have from (2.4) that

$$
\left(\eta_{n}^{2} / 2\right)\left\{1+Q_{n}\left(\eta_{n}\right)\right\} \geqq(3 / 8) \eta_{n}^{2}
$$

so that

$$
\sum \frac{\sigma_{n}^{2}}{s_{n}^{2}} \eta_{n} e^{-(1 / 2) \eta_{n}^{2}\left(1+Q_{n}\left(\eta_{n}\right)\right)}<2 \sum \frac{\sigma_{n}^{2}}{s_{n}^{2}} \frac{\left(\log \log s_{n}\right)^{1 / 2}}{\log ^{3 / 2} s_{n}} .
$$

As $\sum \sigma_{n}^{2}$ diverges, it is seen that the right-hand member of (8.2) is convergent $\left({ }^{17}\right)$. Now, by $(2.25)$, the sequence $\left\{\eta_{n}\right\}$ satisfies the condition of Theorem 1 . Hence $\left\{\eta_{n}\right\} \in \mathcal{V}$.

The convergence of (8.2) implies that the two series

$$
\sum \frac{\sigma_{n}^{2}}{s_{n}^{2}} \phi_{n} \exp \left\{-(1 / 2) \phi_{n}^{2}\left[1+Q_{n}\left(\phi_{n}\right)\right]\right\}
$$

and

$$
\sum \frac{\sigma_{n}^{2}}{s_{n}^{2}} \psi_{n} \exp \left\{-(1 / 2) \psi_{n}^{2}\left[1+Q_{n}\left(\psi_{n}\right)\right]\right\}
$$

either both converge or both diverge: as a matter of fact, those terms in (8.3) which are different from the corresponding terms in (8.4) form a subseries of the left-hand member in (8.2).

On the other hand, if $\left\{\psi_{n}\right\} \in U$, then also $\left\{\phi_{n}\right\} \in U$, since $\psi_{n} \leqq \phi_{n}$. Again, $\psi_{n} \leqq \eta_{n}$ and $\left\{\eta_{n}\right\} \in U$; accordingly, if the inequality $S_{n}>s_{n} \psi_{n}$ is satisfied for infinitely many $n$, this will be the case for such $n$ for which $\psi_{n}=\phi_{n}$. Hence, if $\left\{\psi_{n}\right\} \in \mathcal{L}$, also $\left\{\phi_{n}\right\} \in \mathcal{L}$.

\section{APPENDIX}

The purpose of this appendix is to show how the previous results can be extended, in many cases, to sequences of unbounded variables $X_{n}$. The main application is, of course, to the case where all $X_{n}$ have the same distribution

(17) Cf. Knopp, loc. cit. 
function (cf. the corollary at the end). As a typical example we consider only Theorem 2. The method applies also to the remaining theorems.

From now on the variables $\left\{X_{n}\right\}$ are no longer necessarily bounded; however, we still assume that the second moments (1.3) are finite and that (1.4) holds.

Theorem. Suppose that for some constant $M>0$

$$
\sum_{k} \frac{1}{s_{k}^{2}}\left(\log \log s_{k}\right)^{3} \int_{|x|>M \cdot s_{k} /\left(\log \log s_{k}\right)^{3 / 2}} x^{2} d V_{k}(x) \in \mathcal{C} .
$$

Then $\left\{\phi_{n}\right\} \in \mathcal{U}(\mathcal{L})$ if, and only if,

$$
\sum \frac{\sigma_{n}^{2}}{s_{n}^{2}} \phi_{n} e^{-(1 / 2) \phi_{n}^{2}} \in \mathcal{C}(\mathcal{D}) .
$$

(Needless to say the criterion of Theorem 6 also holds.)

Proof. It follows from the last two paragraphs of $\$ 8$ that it is sufficient to prove the theorem for sequences $\left\{\phi_{n}\right\}$ such that

$$
\phi_{n} \leqq 2\left(\log \log s_{n}\right)^{1 / 2} \text {. }
$$

Put

$$
\begin{gathered}
a_{n}=\int_{|x| \leqq M \cdot s_{n} /\left(\log \log s_{n}\right)^{3 / 2}} x d V_{n}(x) \\
X_{n}^{*}=\left\{\begin{array}{lll}
X_{n}-a_{n} & \text { if } & \left|X_{n}\right| \leqq M s_{n} /\left(\log \log s_{n}\right)^{3 / 2}, \\
a_{n} & \text { if } & \left|X_{n}\right|>M s_{n} /\left(\log \log s_{n}\right)^{3 / 2}
\end{array}\right. \\
X_{n}^{* *}=\left\{\begin{array}{lll}
0 & \text { if } & \left|X_{n}\right| \leqq M s_{n} /\left(\log \log s_{n}\right)^{3 / 2}, \\
X_{n} & \text { if } & \left|X_{n}\right|>M s_{n} /\left(\log \log s_{n}\right)^{3 / 2}
\end{array}\right.
\end{gathered}
$$

Obviously

$$
X_{n}=X_{n}^{*}+X_{n}^{* *}+a_{n}
$$

Now

$$
\begin{aligned}
\sum_{n} \operatorname{Pr}\left(X_{n}^{* *} \neq 0\right) & =\sum_{n} \int_{|x|>M \cdot s_{n} /\left(\log \log s_{n}\right)^{3 / 2}} d V_{n}(x) \\
& \leqq \frac{1}{M^{2}} \sum_{n} \frac{\left(\log \log s_{n}\right)^{3}}{s_{n}^{2}} \int_{|x|>M \cdot s_{n} /\left(\log \log s_{n}\right)^{3 / 2}} x^{2} d V_{n}(x)<\sum_{\infty},
\end{aligned}
$$

so that at almost all points

Next, by (4),

$$
\left|\sum_{n} X_{n}^{* *}\right|=O(1)=o\left(s_{n} / \phi_{n}\right)
$$


(7)

$$
\left|a_{n}\right| \leqq M s_{n} /\left(\log \log s_{n}\right)^{3 / 2},
$$

and therefore by definition

$$
\left|X_{n}^{*}\right| \leqq 2 M s_{n} /\left(\log \log s_{n}\right)^{3 / 2}
$$

The variance of $X_{k}^{*}$ is given by

$$
\begin{aligned}
\sigma_{k}^{* 2} & =\int_{|x| \leqq M \cdot s_{k} /\left(\log \log s_{k}\right)^{3 / 2}}\left(x-a_{k}\right)^{2} d V_{k}(x)+a_{k}^{2} \int_{|x|>M \cdot 8_{k} /\left(\log \log s_{k}\right)^{3 / 2}} d V_{k}(x) \\
& =\int_{|x| \leqq M \cdot s_{k} /\left(\log \log s_{k}\right)^{3 / 2}} x^{2} d V_{k}(x)-a_{k}^{2} \\
& =\sigma_{k}^{2}-\int_{|x|>M \cdot s_{k} /\left(\log \log s_{k}\right)^{3 / 2}} x^{2} d V_{k}(x)-a_{k}^{2} .
\end{aligned}
$$

Now it follows from (1.4) and (1) that

$$
\begin{aligned}
\frac{\left(\log \log s_{n}\right)^{3 / 2}}{s_{n}} \sum_{k=1}^{n}\left|a_{k}\right| & \leqq \sum_{k=1}^{n} \frac{\left(\log \log s_{k}\right)^{3 / 2}}{s_{k}} \int_{|x|>M \cdot s_{k} /\left(\log \log s_{k}\right)^{3 / 2}}|x| d V_{k}(x) \\
& \leqq \frac{1}{M} \sum_{k=1}^{n} \frac{\left(\log \log s_{k}\right)^{3}}{s_{k}^{2}} \int_{|x|>M \cdot s_{k} /\left(\log \log s_{k}\right)^{3 / 2}} x^{2} d V_{k}(x) \\
& =O(1) .
\end{aligned}
$$

Accordingly

$$
\sum_{k=1}^{n}\left|a_{k}\right|=O\left(\frac{s_{n}}{\left(\log \log s_{n}\right)^{3 / 2}}\right)
$$

and, by (7),

$$
\sum_{k=1}^{n}\left|a_{k}^{2}\right|=O\left(\frac{s_{n}^{2}}{\left(\log \log s_{n}\right)^{3}}\right) .
$$

Similarly

$$
\begin{aligned}
\sum_{k=1}^{n} \int_{|x|>M \cdot s_{k} /\left(\log \log s_{k}\right)^{3 / 2}} x^{2} d V_{k}(x) \\
\quad \leqq \frac{s_{n}^{2}}{\left(\log \log s_{n}\right)^{3}} \sum_{k=1}^{n} \frac{\left(\log \log s_{k}\right)^{3}}{s_{k}^{2}} \int_{|x|>M \cdot s_{k} /\left(\log \log s_{k}\right)^{3 / 2}} x^{2} d V_{k}(x) \\
=O\left(\frac{s_{n}^{2}}{\left(\log \log s_{n}\right)^{3}}\right)
\end{aligned}
$$

Combining this with (3), (9), and (11) we have 


$$
s_{n}^{* 2}=\sum_{k=1}^{n} \sigma_{k}^{* 2}=s_{n}^{2}+O\left(\frac{s_{n}^{2}}{\left(\log \log s_{n}\right)^{3}}\right)=s_{n}^{2}+O\left(\frac{s_{n}^{2}}{\phi_{n}^{6}}\right) .
$$

This implies that the two sequences $\left\{\phi_{n}\right\}$ and $\left\{\left(s_{n} / s_{n}^{*}\right) \phi_{n}\right\}$ belong to the same class with respect to $\left\{X_{n}^{*}\right\}$. Now, if $\left\{\phi_{n}\right\} \in \mathcal{L}$, at almost all points the inequality

$$
S_{n}^{*}=\sum_{k=1}^{n} X_{k}^{*}>s_{n}^{*}\left\{\left(s_{n} / s_{n}^{*}\right) \phi_{n}+C\left(s_{n}^{*} / s_{n} \phi_{n}\right)\right\}
$$

will be satisfied for any $C>0$ for infinitely many $n$ so that $\left\{\phi_{n}\right\} \in \mathcal{L}$ also with respect to $\left\{X_{n}\right\}$. A similar remark applies if $\left\{\phi_{n}\right\} \in \mathcal{U}$. In view of (5), (9) the last inequality implies that also $S_{n}>s_{n} \phi_{n}$ for infinitely many $n$. This finishes the proof.

Corollary. The criteria of Theorems 2 and 6 are valid if there exist two constants $\delta>0$ and $A$ such that

$$
\int_{-\infty}^{+\infty} x^{2}|\log | x||^{1+\delta} d V_{k}(x)<A \sigma_{k}^{2}
$$

In particular, this is the case if $V_{k}(x) \equiv V(x)$ and

$$
\int_{-\infty}^{+\infty} x^{2}|\log | x||^{1+\delta} d V(x)<\infty .
$$

In fact, if (14) holds, the series (1) is (at least for sufficiently large $k$ ) majorated by

$$
C \sum_{k} \frac{\sigma_{k}^{2}}{s_{k}^{2} \log ^{1+\delta / 2} s_{k}},
$$

which series obviously converges.

\section{REFERENCES}

1. F. P. Cantelli, Considerazioni sulla legge uniforme dei grandi numeri e sulla generalizzazione di un fondamentale teorema del sig. Paul Léry. Giornale dell'Istituto Italiano degli Attuari vol. 4 (1933) pp. 327-350. 436.

2. P. Erdös, On the law of the iterated logarithm, Ann. of Math. (2) vol 43 (1942) pp. 419-

3. W. Feller, Generalization of a probability limit theorem of Cramer, Trans. Amer. Math. Soc. vol. 54 (1943) pp. 361-372.

4. G. H. Hardy and J. E. Littlewood, Some problems of diophantine approximation, Acta Math. vol. 37 (1914) pp. 155-239. (Cf., in particular, p. 185.)

5. P. Hartman and A. Wintner, On the law of the iterated logarithm, Amer. J. Math. vol. 63 (1941) pp. 169-176.

6. F. Hausdorff, Grundzïge der Mengenlehre, Leipzig, 1913.

7. A. Khintchine, Ueber dyadische Briiche, Math. Zeit. vol. 18 (1923) pp. 109-116.

8. - Ueber einen Satz der Wahrscheinlichkeitsrechnung, Fund. Math. vol. 6 (1924) pp. 9-20. 
9. —_, Ueber das Gesetz der grossen Zahlen, Math. Ann. vol. 96 (1926) pp. 156-168.

10. A. Kolmogoroff, Ueber das Gesetz des iterierten Logarithmus, Math. Ann. vol. 101 (1929) pp. 126-135.

11. P. Lévy, Sur un théorème de M. Khintchine, Bull. Sci. Math. (2) vol. 55 (1931) pp. 145-160.

12. - Théorie de l'addition des variables aléatoires, Paris, Gautier-Villars, 1937.

13. - Sur les séries dont les termes sont des variables éventuelles indépendantes, Studia Mathematica vol. 3 (1931) pp. 117-155.

14. J. Marcinkiewicz, Quelques théorèmes de la théorie des probabilittés, Travaux de la Société des Sciences et des Lettres de Wilno, Classe des Sciences Mathématiques et Naturelles vol. 13 (1939) pp. 1-13.

15. J. Marcinkiewicz and A. Zygmund, Remarque sur la loi du logarithme itêré, Fund. Math. vol. 29 (1937) pp. 215-222.

16. H. Steinhaus, Les probabilités denombrables et leur rapport à la thérie de la mesure, Fund. Math. vol. 4 (1922) pp. 286-310.

17. J. Ville, Etude critique de la notion de collectif, Paris, Gautier-Villars.

Brown UNIVERSITY, Providence, R. I. 